MATHEMATICS OF COMPUTATION

Volume 80, Number 274, April 2011, Pages 697-722

S 0025-5718(2010)02404-4

Article electronically published on August 20, 2010

\title{
SUPERCLOSENESS AND SUPERCONVERGENCE OF STABILIZED LOW-ORDER FINITE ELEMENT DISCRETIZATIONS OF THE STOKES PROBLEM
}

\author{
HAGEN EICHEL, LUTZ TOBISKA, AND HEHU XIE
}

\begin{abstract}
The supercloseness and superconvergence properties of stabilized finite element methods applied to the Stokes problem are studied. We consider consistent residual based stabilization methods as well as inconsistent local projection type stabilizations. Moreover, we are able to show the supercloseness of the linear part of the MINI-element solution which has been previously observed in practical computations. The results on supercloseness hold on three-directional triangular, axiparallel rectangular, and brick-type meshes, respectively, but extensions to more general meshes are also discussed. Applying an appropriate postprocess to the computed solution, we establish superconvergence results. Numerical examples illustrate the theoretical predictions.
\end{abstract}

\section{INTRODUCTION}

In recent years, the superconvergence of finite element methods has been an active research field in numerical analysis. The main objective of the superconvergence research is to improve the existing approximation accuracy by applying certain postprocessing techniques which are cheap and easy to implement.

In this paper, we consider the supercloseness and the superconvergence properties of numerical solutions of the stationary Stokes problem

$$
\left\{\begin{array}{rll}
-\nu \Delta \mathbf{u}+\nabla p= & \mathbf{f} & \text { in } \Omega, \\
\operatorname{div} \mathbf{u}= & 0 & \text { in } \Omega, \\
\mathbf{u} & =0 & \text { on } \partial \Omega .
\end{array}\right.
$$

Here, $\nu>0$ is the kinematic viscosity (we set $\nu=1$ for simplicity) and the function $\mathbf{f}$ is sufficiently smooth. We study finite element approximations on uniform triangular (three-directional grids), axiparallel rectangular, and brick-type meshes, and increase the order of convergence of the original computed solution by postprocessing. The technique for the standard Galerkin finite element approach is wellunderstood; see e.g. [6, 12. If the finite element spaces approximating velocity and pressure satisfy an inf-sup condition, stability and convergence of the discretization can be proven. So far, some superconvergence results have been obtained for the standard Galerkin method; see, for example, [17, 18, 20, 25, 36.

Here, we consider the superconvergence property of stabilized methods which has been developed in order to circumvent the inf-sup condition and to allow equalorder interpolations for velocity and pressure; see [7, 11, 14, 15]. The usual way of

Received by the editor August 3, 2009 and, in revised form, December 10, 2009.

2010 Mathematics Subject Classification. Primary 65N30; Secondary 76D07.

(C)2010 American Mathematical Society 697

Reverts to public domain 28 years from publication 
analyzing superconvergence properties of postprocessed computed solutions consists of two steps:

(1) Supercloseness property: an interpolation approximating the finite element solution of higher order. Often such interpolation does exist if the underlying mesh has a special structure.

(2) A postprocessing operator: an interpolation operator (in a higher-order finite element space) with certain stability, invariance and higher-order approximation properties. Applying this interpolation operator to the original finite element solution, we obtain the postprocessed solution, which has a superconvergence property.

There are many research contributions to these two steps. Currently two approaches are used to prove superconvergence. One is based on the analysis of the method: there are the integral identity, the integral expansion (which is based on the Bramble-Hilbert lemma) and others; see [8, 35. The second concerns the mesh condition 2, 23, 36, 37, 38, 40, 44. The supercloseness result has been extended from structured meshes to more general, practical and automatically generated meshes. In the case of the postprocessing operator, the first approach is manifested as higher-order finite element interpolation, while gradient recovery methods 39, 40, 43, 44 are used in the second approach.

The supercloseness phenomena have been already established for some kinds of mixed finite elements. For example, in [18, 19, 20, 23, 25, the supercloseness analysis for the Stokes problem and Navier-Stokes problems has been given.

For the error analysis, we introduce the standard notation for the Sobolev spaces $W^{k, p}(D), H^{k}(D)=W^{k, 2}(D), H_{0}^{k}(D), L^{p}(D)=W^{0, p}(D)$ with nonnegative integers $k$ and $1 \leq p \leq \infty$. The corresponding vector-valued versions of these spaces will be indicated by boldface letters. The norm and seminorm corresponding to both the scalar and the vector-valued version of the space $W^{k, p}(D)$ are denoted by $\|\cdot\|_{k, p, D}$

and $|\cdot|_{k, p, D}$. For the inner product in $L^{2}(D)$, its vector-valued versions, and $L^{2}(\partial D)$, we write $(\cdot, \cdot)_{D}$ and $\langle\cdot, \cdot\rangle_{\partial D}$, respectively. We will drop the index $D$ when $D=\Omega$. Throughout this paper $C$ denotes a generic positive constant that is independent of the mesh size.

\section{Discretizations of the Stokes Problem}

2.1. Standard Galerkin. Let $\Omega \subset \mathbb{R}^{d}$ be a polygonal $(d=2)$ or polyhedral $(d=3)$ domain with Lipschitz continuous boundary $\Gamma=\partial \Omega$. Introducing the solution spaces $\mathbf{V}:=\left(H_{0}^{1}(\Omega)\right)^{d}$ and $Q:=L_{0}^{2}(\Omega)$, a weak formulation of (1.1) is:

Find $(\mathbf{u}, p) \in \mathbf{V} \times Q$ such that for all $(\mathbf{v}, q) \in \mathbf{V} \times Q$,

$$
(\nabla \mathbf{u}, \nabla \mathbf{v})-(p, \operatorname{div} \mathbf{v})+(q, \operatorname{div} \mathbf{u})=(\mathbf{f}, \mathbf{v}) .
$$

It is well known that the Babuška-Brezzi condition,

$$
\inf _{q \in Q} \sup _{\mathbf{v} \in \mathbf{V}} \frac{(q, \operatorname{div} \mathbf{v})}{|\mathbf{v}|_{1}\|q\|_{0}}>0,
$$

guarantees the existence and uniqueness of a solution of (2.1); cf. [6, 12].

We introduce a shape regular partition $\mathcal{T}_{h}$ of the computational domain $\Omega$ into cells $K$ (triangles, quadrilaterals, tetrahedrons, hexahedrons) such that

$$
\bar{\Omega}=\bigcup_{K \in \mathcal{T}_{h}} \bar{K} .
$$


Here $h:=\max _{K \in \mathcal{T}_{h}}\left\{h_{K}\right\}$ and $h_{K}=\operatorname{diam} K$ denote the global and local mesh size, respectively. Let $\mathbf{V}_{h} \subset \mathbf{V}$ and $Q_{h} \subset Q$ be finite element spaces approximating velocity and pressure. Then, the standard Galerkin discretization is:

Find $\left(\mathbf{u}_{h}, p_{h}\right) \in \mathbf{V}_{h} \times Q_{h}$ such that for all $\left(\mathbf{v}_{h}, q_{h}\right) \in \mathbf{V}_{h} \times Q_{h}$,

$$
\left(\nabla \mathbf{u}_{h}, \nabla \mathbf{v}_{h}\right)-\left(p_{h}, \operatorname{div} \mathbf{v}_{h}\right)+\left(q_{h}, \operatorname{div} \mathbf{u}_{h}\right)=\left(\mathbf{f}, \mathbf{v}_{h}\right) .
$$

The standard Galerkin approach for solving the Stokes problem (1.1) by finite element discretizations is well understood (see, e.g., 6, 12]). If the finite element spaces approximating velocity and pressure satisfy a discrete version of the BabuškaBrezzi condition (2.2) uniformly in $h$, stability and convergence of the discretization can be established. A large number of finite element pairs is known to satisfy this stability condition; however, there are several reasons for circumventing it. First, equal-order interpolations, in general, do not belong to this class of "stable methods", but they are simple to implement since the same finite element space is used for approximating the pressure and the velocity components. Second, and this is even more important, it is often not clear whether the stability property also holds on sequences of meshes with hanging nodes, which are popular in adaptive finite elements. In the case of the $Q_{r}-P_{r-1}^{\text {disc }}$ finite element pair, the validity of the Babuška-Brezzi condition on mesh families with hanging nodes has been shown in 29. Alternative methods for solving the Stokes problem are based on consistent and inconsistent modifications of the discrete problem. These approaches do not require fulfilment of the Babuška-Brezzi condition and work also on families with hanging nodes.

2.2. Local projection stabilization. In this section, we consider equal-order interpolations stabilized by the local projection method in its one-level variant as developed in [11, 26]. For the two-level approach we refer to [3, 5, 27]. Let $Y_{h}$ denote a scalar finite element space of continuous, piecewise polynomials over $\mathcal{T}_{h}$. The spaces for approximating velocity and pressure are given by $\mathbf{V}_{h}:=Y_{h}^{d} \cap \mathbf{V}$ and $Q_{h}:=Y_{h} \cap Q$. The discrete problem of our stabilized method is:

Find $\left(\mathbf{u}_{h}, p_{h}\right) \in \mathbf{V}_{h} \times Q_{h}$ such that for all $\left(\mathbf{v}_{h}, q_{h}\right) \in \mathbf{V}_{h} \times Q_{h}$,

$$
\left(\nabla \mathbf{u}_{h}, \nabla \mathbf{v}_{h}\right)-\left(p_{h}, \operatorname{div} \mathbf{v}_{h}\right)+\left(q_{h}, \operatorname{div} \mathbf{u}_{h}\right)+S_{h}\left(p_{h}, q_{h}\right)=\left(\mathbf{f}, \mathbf{v}_{h}\right),
$$

where the stabilization term with user-chosen parameters $\alpha_{K}$ is given by

$$
S_{h}(p, q)=\sum_{K \in \mathcal{T}_{h}} \alpha_{K}\left(\kappa_{h} \nabla p, \kappa_{h} \nabla q\right)_{K}
$$

Here, the fluctuation operator $\kappa_{h}: L^{2}(\Omega) \rightarrow L^{2}(\Omega)$ acting componentwise is defined as follows. Let $P_{s}(K)$ denote the set of all polynomials of degree less than or equal to $s$ and let $D_{h}(K)$ be a finite-dimensional space on the cell $K \in \mathcal{T}_{h}$ with $P_{s}(K) \subset D_{h}(K)$. We extend the definition by allowing $P_{-1}(K)=D_{h}(K)=\{0\}$. We introduce the associated global space of discontinuous finite elements

$$
D_{h}:=\bigoplus_{K \in \mathcal{T}_{h}} D_{h}(K)
$$

and the local $L^{2}(K)$-projection $\pi_{K}: L^{2}(K) \rightarrow D_{h}(K)$ generating the global projection $\pi_{h}: L^{2}(\Omega) \rightarrow D_{h}$ by

$$
\left.\left(\pi_{h} w\right)\right|_{K}:=\pi_{K}\left(\left.w\right|_{K}\right) \quad \forall K \in \mathcal{T}_{h}, \forall w \in L^{2}(\Omega) .
$$


The fluctuation operator $\kappa_{h}: L^{2}(\Omega) \rightarrow L^{2}(\Omega)$ used in (2.5) is given by $\kappa_{h}:=i d-\pi_{h}$, where $i d: L^{2}(\Omega) \rightarrow L^{2}(\Omega)$ is the identity on $L^{2}(\Omega)$.

In order to study the supercloseness and superconvergence properties of this method on structured meshes, we introduce the bilinear form

$$
A_{h}((\mathbf{u}, p) ;(\mathbf{v}, q))=(\nabla \mathbf{u}, \nabla \mathbf{v})-(p, \operatorname{div} \mathbf{v})+(q, \operatorname{div} \mathbf{u})+S_{h}(p, q)
$$

and the mesh-dependent norm

$$
\||(\mathbf{v}, q)|\|_{A}:=\left(|\mathbf{v}|_{1}^{2}+\|q\|_{0}^{2}+\sum_{K \in \mathcal{T}_{h}} \alpha_{K}\left\|\kappa_{h} \nabla q\right\|_{0, K}^{2}\right)^{1 / 2} .
$$

From (2.1) and (2.4) follows the error equation

$$
A_{h}\left(\left(\mathbf{u}-\mathbf{u}_{h}, p-p_{h}\right),\left(\mathbf{v}_{h}, q_{h}\right)\right)=S_{h}\left(p, q_{h}\right) \quad \forall\left(\mathbf{v}_{h}, q_{h}\right) \in \mathbf{V}_{h} \times Q_{h},
$$

which shows that in contrast to residual based stabilization methods [14, 15, the method is inconsistent.

The existence and uniqueness of discrete solutions of (2.4) have been studied in 11 for different pairs $\left(Y_{h}, D_{h}\right)$ of approximation and projection spaces, respectively. Here, the supercloseness and superconvergence properties will be studied only for the lowest-order cases; i.e., we will consider

- on three-directional triangular meshes $(d=2)$ the cases

$$
\begin{aligned}
& Y_{h}:=\left\{v \in H^{1}(\Omega):\left.v\right|_{K} \in P_{1}(K), \forall K \in \mathcal{T}_{h}\right\}, D_{h}:=\{0\}, \alpha_{K} \sim h_{K}^{2}, \\
& Y_{h}:=\left\{v \in H^{1}(\Omega):\left.v\right|_{K} \in P_{1}^{+}(K), \forall K \in \mathcal{T}_{h}\right\}, \\
& D_{h}:=\left\{q \in L^{2}(\Omega):\left.q\right|_{K} \in P_{0}(K), \forall K \in \mathcal{T}_{h}\right\}, \alpha_{K} \sim h_{K} \text { or } \alpha_{K} \sim h_{K}^{2},
\end{aligned}
$$

- on rectangular or brick meshes $(d=2$ or $d=3)$ the case

$$
Y_{h}:=\left\{v \in H^{1}(\Omega):\left.v\right|_{K} \in Q_{1}(K), \forall K \in \mathcal{T}_{h}\right\}, D_{h}:=\{0\}, \alpha_{K} \sim h_{K}^{2},
$$

where $Q_{1}(K)$ denotes the space of mapped bilinear and trilinear functions, respectively, and $P_{1}^{+}(K)$ is the space of linear functions enriched by cubic bubbles vanishing on the boundary of $K$. In the following, we will refer to these different cases shortly as the $P_{1}$, the $P_{1}^{+}$, and the $Q_{1}$ case, respectively.

All three cases fit to the theory developed in [11]; consequently we have the following stability and convergence result.

Lemma 2.1 ([1]). Assume $h_{K}^{2} / \alpha_{K} \leq C$. Then, there is a positive constant $\beta_{A}$ independent of $h$ such that

$$
\inf _{\left(\mathbf{v}_{h}, q_{h}\right) \in \mathbf{V}_{h} \times Q_{h}} \sup _{\left(\mathbf{w}_{h}, r_{h}\right) \in \mathbf{V}_{h} \times Q_{h}} \frac{A_{h}\left(\left(\mathbf{v}_{h}, q_{h}\right) ;\left(\mathbf{w}_{h}, r_{h}\right)\right)}{\left\|\left(\mathbf{v}_{h}, q_{h}\right)\right\|\left\|_{A} \mid\right\|\left(\mathbf{w}_{h}, r_{h}\right)\|\|_{A}} \geq \beta_{A}>0
$$

holds.

Lemma 2.2 ([11). Let the solution $(\mathbf{u}, p)$ of (2.1) belong to $\left(\mathbf{V} \cap H^{2}(\Omega)^{d}\right) \times(Q \cap$ $\left.H^{1}(\Omega)\right)$. Then, there exists a positive constant $C$ independent of $h$ such that the solution $\left(\mathbf{u}_{h}, p_{h}\right)$ of (2.4) satisfies

$$
\text { ||l(u- } \left.\mathbf{u}_{h}, p-p_{h}\right)||_{A} \leq C h\left(\|\mathbf{u}\|_{2}+\|p\|_{1}\right) .
$$

Remark 2.3. In the two cases in which $D_{h}=\{0\}$, the fluctuation operator becomes the identity and (2.4) corresponds to the method studied in [7]. 
2.3. Relationship to residual based stabilizations. In the case of $Y_{h}=\{v \in$ $\left.H^{1}(\Omega):\left.v\right|_{K} \in P_{1}^{+}(K), \forall K \in \mathcal{T}_{h}\right\}$, the standard space of continuous, piecewise linear functions has been enriched by one cubic bubble function per cell. These additional degrees of freedom can be eliminated locally by static condensation [11, Section 4.3]. Based on the splitting of the approximation space $Y_{h}=Y_{L} \oplus Y_{B}$ into the piecewise linear part $Y_{L}$ and the bubble part $Y_{B}$ the solution $\left(\mathbf{u}_{h}, p_{h}\right)$ of (2.4) can be split into $\mathbf{u}_{h}=\mathbf{u}_{L}+\mathbf{u}_{B}$ and $p_{h}=\tilde{p}_{L}+p_{B}$ with $\mathbf{u}_{L} \in \mathbf{V}_{L}=Y_{L}^{2} \cap \mathbf{V}$ and $\tilde{p}_{L} \in Y_{L}$. Let us define

$$
p_{L}=\tilde{p}_{L}-\frac{1}{|\Omega|} \int_{\Omega} \tilde{p}_{L} d x \in Q_{L}:=Y_{L} \cap Q .
$$

Then, as shown in [11, the linear part $\left(\mathbf{u}_{L}, p_{L}\right) \in \mathbf{V}_{L} \times Q_{L}$ of $\left(\mathbf{u}_{h}, p_{h}\right) \in \mathbf{V}_{h} \times Q_{h}$ is a solution of the problem:

Find $\left(\mathbf{u}_{L}, p_{L}\right) \in \mathbf{V}_{L} \times Q_{L}$ such that for all $\left(\mathbf{v}_{L}, q_{L}\right) \in \mathbf{V}_{L} \times Q_{L}$,

$$
B_{h}\left(\left(\mathbf{u}_{L}, p_{L}\right) ;\left(\mathbf{v}_{L}, q_{L}\right)\right)=L_{h}\left(\mathbf{v}_{L}, q_{L}\right) .
$$

The bilinear form $B_{h}(\cdot ; \cdot)$ and the linear form $L_{h}(\cdot)$ are defined by

$$
\begin{aligned}
& B_{h}((\mathbf{u}, p) ;(\mathbf{v}, q)):=(\nabla \mathbf{u}, \nabla \mathbf{v})-(p, \operatorname{div} \mathbf{v})+(q, \operatorname{div} \mathbf{u}) \\
&+\sum_{K \in \mathcal{T}_{h}} \gamma_{K}(\operatorname{div} \mathbf{u}, \operatorname{div} \mathbf{v})_{K}+\sum_{K \in \mathcal{T}_{h}}\left(-\Delta \mathbf{u}+\nabla p, \tau_{K} \nabla q\right)_{K}, \\
& L_{h}(\mathbf{v}, q):=(\mathbf{f}, \mathbf{v})+\sum_{K \in \mathcal{T}_{h}}\left(\mathbf{f}, \tau_{K} \nabla q\right)_{K},
\end{aligned}
$$

with

$$
\gamma_{K}=\frac{\left\|b_{K}\right\|_{0,1, K}^{2}}{\alpha_{K}|K|\left\|\kappa_{h} \nabla b_{K}\right\|_{0, K}^{2}}, \quad \tau_{K}=\frac{\left\|b_{K}\right\|_{0,1, K}}{\left|b_{K}\right|_{1, K}^{2}} b_{K}, \quad b_{K}=\lambda_{1} \lambda_{2} \lambda_{3} .
$$

Here, $\lambda_{i}, i=1,2,3$, denote the barycentric coordinates of $K$. Note that

$$
\tau_{K} \sim h_{K}^{2} b_{K}
$$

and that, depending on the choice of the stabilization parameter $\alpha_{K}$ (which is related to the approximation space $D_{h}$ ) in the LPS, we have

$$
\alpha_{K} \sim h_{K}^{2} \Leftrightarrow \gamma_{K} \sim 1, \quad \alpha_{K} \sim h_{K} \Leftrightarrow \gamma_{K} \sim h_{K} .
$$

We mention that the problem (2.11) corresponds to the Pressure Stabilized Petrov Galerkin (PSPG) method [14, 15, 31] in combination with the grad-div stabilization [10, 13, 32, 33. The PSPG stabilization is consistent in the sense that for a smooth solution $(\mathbf{u}, p) \in\left(H_{0}^{1}(\Omega) \cap H^{2}(\Omega)\right)^{d} \times\left(L_{0}^{2}(\Omega) \cap H^{1}(\Omega)\right)$,

$$
B_{h}\left((\mathbf{u}, p) ;\left(\mathbf{v}_{L}, q_{L}\right)\right)=L_{h}\left(\mathbf{v}_{L}, q_{L}\right) \quad \forall\left(\mathbf{v}_{L}, q_{L}\right) \in \mathbf{V}_{L} \times Q_{L}
$$

holds. We have two options for analyzing the method (2.11): using the error estimate (2.10) and deriving estimates for the linear part of the solution, or studying directly the PSPG method (2.11). We follow the second option by proving the stability of the bilinear form $B_{h}:\left(\mathbf{V}_{L} \times Q_{L}\right) \times\left(\mathbf{V}_{L} \times Q_{L}\right) \rightarrow \mathbb{R}$ with respect to the mesh-dependent norm

$$
\|\mid(\mathbf{v}, q)\| \|_{B}:=\left(|\mathbf{v}|_{1}^{2}+\|q\|_{0}^{2}+\sum_{K \in \mathcal{T}_{h}} \gamma_{K}\|\operatorname{div} \mathbf{v}\|_{0, K}^{2}+\sum_{K \in \mathcal{T}_{h}}\left\|\tau_{K}^{1 / 2} \nabla q\right\|_{0, K}^{2}\right)^{1 / 2} .
$$


Lemma 2.4. Assume $\gamma_{K}=\mathcal{O}(1)$ and $\tau_{K} \sim h_{K}^{2} b_{K}$. Then, there is a positive constant $\beta_{B}$ independent of $h$ such that

$$
\inf _{\left(\mathbf{v}_{L}, q_{L}\right) \in \mathbf{V}_{L} \times Q_{L}} \sup _{\left(\mathbf{w}_{L}, r_{L}\right) \in \mathbf{V}_{L} \times Q_{L}} \frac{B_{h}\left(\left(\mathbf{v}_{L}, q_{L}\right) ;\left(\mathbf{w}_{L}, r_{L}\right)\right)}{\left\|\left(\mathbf{v}_{L}, q_{L}\right)\right\|\left\|_{B} \mid\right\|\left(\mathbf{w}_{L}, r_{L}\right)\|\|_{B}} \geq \beta_{B}>0
$$

holds.

Proof. Let $\left(\mathbf{v}_{L}, q_{L}\right)$ be an arbitrary element of $\mathbf{V}_{L} \times Q_{L}$. We obtain

$$
B_{h}\left(\left(\mathbf{v}_{L}, q_{L}\right) ;\left(\mathbf{v}_{L}, q_{L}\right)\right)=\left|\mathbf{v}_{L}\right|_{1}^{2}+\sum_{K \in \mathcal{T}_{h}} \gamma_{K}\left\|\operatorname{div} \mathbf{v}_{L}\right\|_{0, K}^{2}+\sum_{K \in \mathcal{T}_{h}}\left\|\tau_{K}^{1 / 2} \nabla q_{L}\right\|_{0, K}^{2}
$$

Compared with (2.12), just the $L^{2}$ control over the pressure is missing. Due to the continuous inf-sup condition (2.2) there is for any $q_{L} \in Q_{L}$ an element $\mathbf{v}_{q_{L}} \in \mathbf{V}$ satisfying

$$
-\left(q_{L}, \operatorname{div} \mathbf{v}_{q_{L}}\right)=\left\|q_{L}\right\|_{0}^{2}, \quad\left\|\mathbf{v}_{q_{L}}\right\|_{1} \leq C\left\|q_{L}\right\|_{0} .
$$

As a consequence, we have for the Scott-Zhang [30] interpolant $i_{h}: H_{0}^{1}(\Omega)^{2} \rightarrow \mathbf{V}_{L}$,

$$
\begin{aligned}
& B_{h}\left(\left(\mathbf{v}_{L}, q_{L}\right) ;\left(i_{h} \mathbf{v}_{q_{L}}, 0\right)\right)=-\left(q_{L}, \operatorname{div}\right.\left.i_{h} \mathbf{v}_{q_{L}}\right)+\left(\nabla \mathbf{v}_{L}, \nabla i_{h} \mathbf{v}_{q_{L}}\right) \\
&+\sum_{K \in \mathcal{T}_{h}} \gamma_{K}\left(\operatorname{div} \mathbf{v}_{L}, \operatorname{div} i_{h} \mathbf{v}_{q_{L}}\right)_{K} \\
&=\left\|q_{L}\right\|_{0}^{2}+\left(q_{L}, \operatorname{div}\left(\mathbf{v}_{q_{L}}-i_{h} \mathbf{v}_{q_{L}}\right)\right)+\left(\nabla \mathbf{v}_{L}, \nabla i_{h} \mathbf{v}_{q_{L}}\right) \\
&+\sum_{K \in \mathcal{T}_{h}} \gamma_{K}\left(\operatorname{div} \mathbf{v}_{L}, \operatorname{div} i_{h} \mathbf{v}_{q_{L}}\right)_{K} .
\end{aligned}
$$

Using integration by parts, the approximation property of the Scott-Zhang [30] interpolation

$$
\left\|\mathbf{v}-i_{h} \mathbf{v}\right\|_{0, K} \leq C h_{K}\|\mathbf{v}\|_{1, \omega(K)} \quad \forall \mathbf{v} \in H^{1}(K), K \in \mathcal{T}_{h},
$$

the inequality

$$
\left\|\tau_{K}^{1 / 2} \nabla q_{L}\right\|_{0, K} \geq C h_{K}\left\|\nabla q_{L}\right\|_{0, K} \quad \forall q_{L} \in P_{1}(K),
$$

and the bound of $\left\|\mathbf{v}_{q_{L}}\right\|_{1}$, we estimate the second term in (2.15) as follows:

$$
\begin{aligned}
\left|\left(q_{L}, \operatorname{div}\left(\mathbf{v}_{q_{L}}-i_{h} \mathbf{v}_{q_{L}}\right)\right)\right| & =\left|\left(\nabla q_{L}, \mathbf{v}_{q_{L}}-i_{h} \mathbf{v}_{q_{L}}\right)\right| \leq C \sum_{K \in \mathcal{T}_{h}}\left\|\nabla q_{L}\right\|_{0, K} h_{K}\left\|\mathbf{v}_{q_{L}}\right\|_{1, \omega(K)} \\
& \leq C\left(\sum_{K \in \mathcal{T}_{h}}\left\|\tau_{K}^{1 / 2} \nabla q_{L}\right\|_{0, K}^{2}\right)^{1 / 2}\left(\sum_{K \in \mathcal{T}_{h}}\left\|\mathbf{v}_{q_{L}}\right\|_{1, \omega(K)}^{2}\right)^{1 / 2} \\
& \leq \frac{1}{6}\left\|q_{L}\right\|_{0}^{2}+C_{1} \sum_{K \in \mathcal{T}_{h}}\left\|\tau_{K}^{1 / 2} \nabla q_{L}\right\|_{0, K}^{2} .
\end{aligned}
$$

The estimations of the third and fourth terms in (2.15) are standard:

$$
\begin{aligned}
\left|\left(\nabla \mathbf{v}_{L}, \nabla i_{h} \mathbf{v}_{q_{L}}\right)\right| & \leq C\left|\nabla \mathbf{v}_{L}\right|_{1}\left|\nabla \mathbf{v}_{q_{L}}\right|_{1} \leq \frac{1}{6}\left\|q_{L}\right\|_{0}^{2}+C_{2}\left|\nabla \mathbf{v}_{L}\right|_{1}^{2}, \\
\left|\sum_{K \in \mathcal{T}_{h}} \gamma_{K}\left(\operatorname{div} \mathbf{v}_{L}, \operatorname{div} i_{h} \mathbf{v}_{q_{L}}\right)_{K}\right| & \leq \frac{1}{6}\left\|q_{L}\right\|_{0}^{2}+C_{3} \sum_{K \in \mathcal{T}_{h}} \gamma_{K}\left\|\operatorname{div} \mathbf{v}_{L}\right\|_{0, K}^{2} .
\end{aligned}
$$


Summing up the last three inequalities, we obtain from (2.15),

$$
\begin{aligned}
& B_{h}\left(\left(\mathbf{v}_{L}, q_{L}\right) ;\left(i_{h} \mathbf{v}_{q_{L}}, 0\right)\right) \\
& \quad \geq \frac{1}{2}\left\|q_{L}\right\|_{0}^{2}-C_{4}\left(\left|\nabla \mathbf{v}_{L}\right|_{1}^{2}+\sum_{K \in \mathcal{T}_{h}}\left[\left\|\tau_{K}^{1 / 2} \nabla q_{L}\right\|_{0, K}^{2}+\gamma_{K}\left\|\operatorname{div} \mathbf{v}_{L}\right\|_{0, K}^{2}\right]\right)
\end{aligned}
$$

with $C_{4}=\max \left(C_{1}, C_{2}, C_{3}\right)$. Multiplying this inequality by $2 /\left(1+2 C_{4}\right)$ and adding it to (2.14), we see that for any $\left(\mathbf{v}_{L}, q_{L}\right) \in \mathbf{V}_{L} \times Q_{L}$, there exists

$$
\left(\mathbf{w}_{L}, r_{L}\right):=\left(\mathbf{v}_{L}, q_{L}\right)+\left(2 /\left(1+2 C_{4}\right)\right)\left(i_{h} \mathbf{v}_{q_{L}}, 0\right)
$$

such that

$$
B_{h}\left(\left(\mathbf{v}_{L}, q_{L}\right) ;\left(\mathbf{w}_{L}, r_{L}\right)\right) \geq \frac{1}{1+2 C_{4}}\left\|\left(\mathbf{v}_{L}, q_{L}\right) \mid\right\|_{B}^{2} .
$$

Furthermore, the $H^{1}$-stability of the interpolation $i_{h}$, the upper bound of $\left\|\mathbf{v}_{q_{L}}\right\|_{1}$, and $\gamma_{K} \leq \gamma_{0}$ lead to

$$
\left\|\left|\left(\mathbf{w}_{L}, r_{L}\right)\right|\right\|_{B} \leq\left\|| | ( \mathbf { v } _ { L } , q _ { L } ) \left|\left\|_{B}+\frac{2}{1+2 C_{4}}\left|\left\|( i _ { h } \mathbf { v } _ { L } , 0 ) \left|\left\|_{B} \leq\left(1+C_{5}\right) \mid\right\|\left(\mathbf{v}_{L}, q_{L}\right)\|\|_{B} .\right.\right.\right.\right.\right.\right.
$$

Thus, the statement of the lemma holds true with $\beta=1 /\left(\left(1+2 C_{4}\right)\left(1+C_{5}\right)\right)$.

Taking into consideration the approximation properties of the space $\mathbf{V}_{L} \times Q_{L}$ we get

Lemma 2.5. Assume $\gamma_{K}=\mathcal{O}(1)$ and $\tau_{K} \sim h_{K}^{2} b_{K}$. Let the solution $(\mathbf{u}, p)$ of (2.1) belong to $\left(\mathbf{V} \cap H^{2}(\Omega)^{d}\right) \times\left(Q \cap H^{1}(\Omega)\right)$. Then, there exists a positive constant $C$ independent of $h$ such that the solution $\left(\mathbf{u}_{L}, p_{L}\right)$ of (2.11) satisfies

$$
\left\|\left|\left(\mathbf{u}-\mathbf{u}_{L}, p-p_{L}\right)\right|\right\|_{B} \leq C h\left(\|\mathbf{u}\|_{2}+\|p\|_{1}\right) .
$$

Finally in this section, we mention the relationship of the standard Galerkin discretization using the MINI-element to the residual based stabilization method (2.11). In this case, the velocity and pressure are approximated by elements from the spaces

$$
\begin{aligned}
& \mathbf{V}_{h}=\mathbf{V}_{L}^{+}=\left\{\mathbf{v} \in H_{0}^{1}(\Omega)^{d}:\left.\mathbf{v}\right|_{K} \in P_{1}^{+}(K)^{d}, \forall K \in \mathcal{T}_{h}\right\}, \\
& Q_{h}=Q_{L}=\left\{q \in L_{0}^{2}(\Omega) \cap H^{1}(\Omega):\left.q\right|_{K} \in P_{1}(K) \forall K \in \mathcal{T}_{h}\right\} .
\end{aligned}
$$

Note that the MINI-element satisfies the discrete version of (2.2); see [1]. Thus no stabilization term is needed. Again eliminating the bubble part in the velocity space we end up with the method (2.11) in $\mathbf{V}_{L} \times Q_{L}$ for $\gamma_{K}=0$. This relationship between the MINI-element discretization and the residual based stabilization will allow us to prove supercloseness results for the linear part of the MINI-element discretization.

\section{Supercloseness}

In this section, we consider structured meshes, in particular three-directional triangular meshes in the two-dimensional case and uniform brick meshes in the three-dimensional case. All theorems for the brick meshes hold analogously also for rectangular meshes; however, we do not formulate them explicitly. 
3.1. Piecewise linear interpolations on three-directional meshes. We start with some interpolation error estimates which are necessary for our supercloseness analysis. Let $i_{h}: H^{2}(\Omega)^{2} \rightarrow \mathbb{R}^{2}$ and $j_{h}: H^{2}(\Omega) \rightarrow \mathbb{R}$ denote the standard piecewise linear nodal interpolation. In order to derive the supercloseness property of $\left(\mathbf{u}_{h}, p_{h}\right)$ to $\left(i_{h} \mathbf{u}, j_{h} p\right)$, we recall some estimates which can be found, e.g., in [22] and in the books [19, 24].

Lemma 3.1 ([19, 22, 24]). Let $\mathbf{u} \in H^{3}(\Omega)^{2}$ and the mesh $\mathcal{T}_{h}$ be three-directional. Then, we have the estimate

$$
\left|\left(\nabla\left(\mathbf{u}-i_{h} \mathbf{u}\right), \nabla \mathbf{w}_{h}\right)\right| \leq C h^{2}\|\mathbf{u}\|_{3}\left|\mathbf{w}_{h}\right|_{1}, \quad \forall \mathbf{w}_{h} \in \mathbf{V}_{h} .
$$

Remark 3.2. The estimate (3.1) has been obtained by many researchers and may be the oldest supercloseness result $(28])$. Nowadays, the proof of this estimate has been simplified and extended to more general meshes [2], which we consider in Section 5 .

Let us define the notation illustrated in Figure 1. For an arbitrary triangle

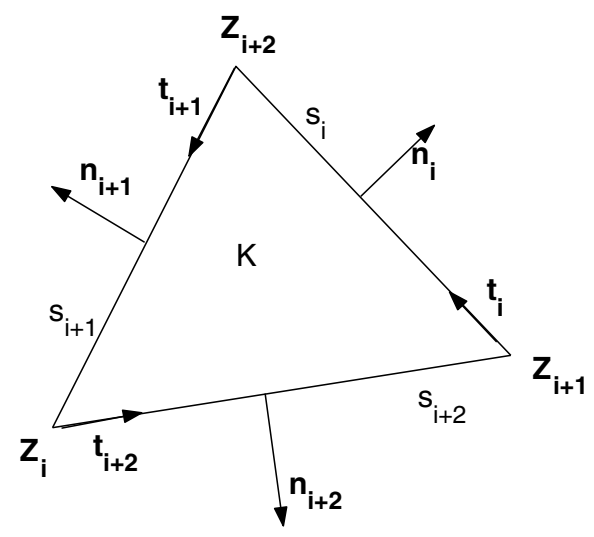

FIGURE 1. Some notation of a triangle $K \in \mathcal{T}_{h}$.

$K \in \mathcal{T}_{h}$, let $Z_{i}=\left(x_{i 1}, x_{i 2}\right)(1 \leq i \leq 3)$ be the counterclockwise oriented vertices, $s_{i}$ $(1 \leq i \leq 3)$ denote the edge of length $h_{i}(1 \leq i \leq 3)$ opposite to $Z_{i} ; \mathbf{n}_{i}(1 \leq i \leq 3)$ is the unit outward normal vector on $s_{i}$, and $\mathbf{t}_{i}(1 \leq i \leq 3)$ are the unit tangent vectors in the counterclockwise orientation. We use the periodic relation for the subscripts: $i+3=i$ and write for the derivative in the direction of $\mathbf{t}_{i}$ shortly $\partial_{\mathbf{t}_{i}}=\partial / \partial \mathbf{t}_{i}$. As usual, let the reference triangle $\widehat{K}$ have the vertices $(0,0),(1,0)$, and $(0,1)$.

Lemma 3.3. Let $\widehat{I}$ be the standard piecewise linear nodal interpolation on $\widehat{K}$. Then, there are positive constants $C$ such that for all $\hat{\varphi} \in H^{3}(\widehat{K})$ and for all $\hat{\psi} \in P_{0}(\widehat{K})$,

$$
\left|\int_{\widehat{K}}(\hat{\varphi}-\widehat{I} \hat{\varphi}) \hat{\psi} d \hat{x}+\frac{1}{12} \int_{\widehat{K}}\left(\hat{\varphi}_{\hat{x}_{1} \hat{x}_{1}}-\hat{\varphi}_{\hat{x}_{1} \hat{x}_{2}}+\hat{\varphi}_{\hat{x}_{2} \hat{x}_{2}}\right) \hat{\psi} d \hat{x}\right| \leq C|\hat{\varphi}|_{3, \widehat{K}}\|\hat{\psi}\|_{0, \widehat{K}} \text {. }
$$

Proof. We use a Bramble-Hilbert type argument [9, Theorem 4.1.3] in order to prove the expansion formulas on the reference element $\widehat{K}$. For fixed $\hat{\psi} \in P_{0}(\widehat{K})$, we consider the following continuous linear form $\Phi: H^{3}(\widehat{K}) \rightarrow \mathbb{R}$ given by

$$
\hat{\varphi} \mapsto \Phi(\hat{\varphi})=\int_{\widehat{K}}(\hat{\varphi}-\widehat{I} \hat{\varphi}) \hat{\psi} d \hat{x}+\frac{1}{12} \int_{\widehat{K}}\left(\hat{\varphi}_{\hat{x}_{1} \hat{x}_{1}}-\hat{\varphi}_{\hat{x}_{1} \hat{x}_{2}}+\hat{\varphi}_{\hat{x}_{2} \hat{x}_{2}}\right) \hat{\psi} d \hat{x}
$$


for which

$$
|\Phi(\hat{\varphi})| \leq C\|\hat{\varphi}\|_{3, \widehat{K}}\|\hat{\psi}\|_{0, \widehat{K}}
$$

holds true. When $\hat{\varphi}$ equals $\hat{x}_{1}^{2}, \hat{x}_{1} \hat{x}_{2}$, and $\hat{x}_{2}^{2}$, respectively, the corresponding interpolations $\widehat{I} \hat{u}$ become $\hat{x}_{1}, 0$, and $\hat{x}_{2}$. A direct computation shows that

$$
\Phi(\hat{\varphi})=0, \quad \forall \hat{\varphi} \in P_{2}(\widehat{K})
$$

Consequently, there is a positive constant $C$ such that (3.2) holds true.

Lemma 3.4. Let $\mathbf{u} \in H^{3}(\Omega)^{2} \cap \mathbf{V}$ and the mesh $\mathcal{T}_{h}$ be three-directional. Then, we have the estimates

$$
\begin{array}{rlrl}
\left|\left(r_{h}, \operatorname{div}\left(\mathbf{u}-i_{h} \mathbf{u}\right)\right)\right| & \leq C h^{3 / 2}\|\mathbf{u}\|_{3}\left\|r_{h}\right\|_{0}, & & \forall r_{h} \in Q_{h}, \\
\left|\left(\operatorname{div}\left(\mathbf{u}-i_{h} \mathbf{u}\right), \operatorname{div} \mathbf{v}_{h}\right)\right| \leq C h^{2}\|\mathbf{u}\|_{3}\left\|\mathbf{v}_{h}\right\|_{1}, & \forall \mathbf{v}_{h} \in \mathbf{V}_{h} .
\end{array}
$$

Proof. We use techniques similar to [41, 42]. We start with (3.3), integrate by parts

$$
\left(r_{h}, \operatorname{div}\left(\mathbf{u}-i_{h} \mathbf{u}\right)\right)=-\left(\mathbf{u}-i_{h} \mathbf{u}, \nabla r_{h}\right)=-\sum_{K \in \mathcal{T}_{h}}\left(\mathbf{u}-i_{h} \mathbf{u}, \nabla r_{h}\right)_{K},
$$

and define an affine mapping $F_{K}: \widehat{K} \rightarrow K$ by

$$
x=F_{K} \hat{x}=\left(h_{1} \mathbf{t}_{1},-h_{3} \mathbf{t}_{3}\right) \cdot \hat{x}+\mathbf{Z}_{2}=B_{K} \hat{x}+\mathbf{Z}_{2} .
$$

Then, for a function $\hat{w}: \widehat{K} \rightarrow \mathbb{R}$ and $w=\hat{w} \circ F_{K}^{-1}$ we have

$$
\begin{gathered}
\hat{w}_{\hat{x}_{1}}=h_{1}\left(\partial_{\mathbf{t}_{1}} w\right) \circ F_{K}, \quad \hat{w}_{\hat{x}_{2}}=-h_{3}\left(\partial_{\mathbf{t}_{3}} w\right) \circ F_{K}, \\
\hat{w}_{\hat{x}_{1} \hat{x}_{1}}=h_{1}^{2}\left(\partial_{\mathbf{t}_{1} \mathbf{t}_{1}}^{2} w\right) \circ F_{K}, \quad \hat{w}_{\hat{x}_{1} \hat{x}_{2}}=-h_{1} h_{3}\left(\partial_{\mathbf{t}_{1} \mathbf{t}_{3}}^{2} w\right) \circ F_{K}, \quad \hat{w}_{\hat{x}_{2} \hat{x}_{2}}=h_{3}^{2}\left(\partial_{\mathbf{t}_{3} \mathbf{t}_{3}}^{2} w\right) \circ F_{K} .
\end{gathered}
$$

Now, transforming onto the reference triangle $\widehat{K}$, using Lemma 3.3 componentwise, transforming back to the original element $K$, and integrating by parts, we get

$$
\begin{aligned}
\int_{K}\left(\mathbf{u}-i_{h} \mathbf{u}\right) \nabla r_{h} d x= & \operatorname{det} B_{K} \int_{\widehat{K}}(\hat{\mathbf{u}}-\widehat{I} \hat{\mathbf{u}}) B_{K}^{-T} \hat{\nabla} \hat{r}_{h} d \hat{x} \\
= & -\frac{\operatorname{det} B_{K}}{12} \int_{\widehat{K}}\left(\hat{\mathbf{u}}_{\hat{x}_{1} \hat{x}_{1}}-\hat{\mathbf{u}}_{\hat{x}_{1} \hat{x}_{2}}+\hat{\mathbf{u}}_{\hat{x}_{2} \hat{x}_{2}}\right) B_{K}^{-T} \hat{\nabla} \hat{r}_{h} d \hat{x}+R \\
= & -\frac{1}{12} \int_{K}\left(h_{1}^{2} \partial_{\mathbf{t}_{1} \mathbf{t}_{1}}^{2} \mathbf{u}+h_{1} h_{3} \partial_{\mathbf{t}_{1} \mathbf{t}_{3}}^{2} \mathbf{u}+h_{3}^{2} \partial_{\mathbf{t}_{3} \mathbf{t}_{3}}^{2} \mathbf{u}\right) \nabla r_{h} d x+R \\
= & \frac{1}{12} \int_{K} r_{h} \operatorname{div}\left(h_{1}^{2} \partial_{\mathbf{t}_{1} \mathbf{t}_{1}}^{2} \mathbf{u}+h_{1} h_{3} \partial_{\mathbf{t}_{1} \mathbf{t}_{3}}^{2} \mathbf{u}+h_{3}^{2} \partial_{\mathbf{t}_{3} \mathbf{t}_{3}}^{2} \mathbf{u}\right) d x+R \\
& -\frac{1}{12} \int_{\partial K} r_{h}\left(h_{1}^{2} \partial_{\mathbf{t}_{1} \mathbf{t}_{1}}^{2} \mathbf{u}+h_{1} h_{3} \partial_{\mathbf{t}_{1} \mathbf{t}_{3}}^{2} \mathbf{u}+h_{3}^{2} \partial_{\mathbf{t}_{3} \mathbf{t}_{3}}^{2} \mathbf{u}\right) \cdot \mathbf{n}_{K} d s
\end{aligned}
$$

The first term can be estimated by the Cauchy-Schwarz inequality, leading to

$$
\left|\frac{1}{12} \int_{K} r_{h} \operatorname{div}\left(h_{1}^{2} \partial_{\mathbf{t}_{1} \mathbf{t}_{1}}^{2} \mathbf{u}+h_{1} h_{3} \partial_{\mathbf{t}_{1} \mathbf{t}_{3}}^{2} \mathbf{u}+h_{3}^{2} \partial_{\mathbf{t}_{3} \mathbf{t}_{3}}^{2} \mathbf{u}\right) d x\right| \leq C h_{K}^{2}|\mathbf{u}|_{3, K}\left\|r_{h}\right\|_{0, K},
$$

and for the second we obtain from Lemma 3.3

$|R| \leq C \operatorname{det} B_{K}|\hat{\mathbf{u}}|_{3, \widehat{K}}\left\|B_{K}^{-T} \hat{\nabla} \hat{r}_{h}\right\|_{0, \widehat{K}} \leq C\left\|B_{K}\right\|^{3}|\mathbf{u}|_{3, K}\left|r_{h}\right|_{1, K} \leq C h_{K}^{2}|\mathbf{u}|_{3, K}\left\|r_{h}\right\|_{0, K}$,

where, in the last step, we used an inverse estimate and the standard estimates for affine-equivalent finite elements [9, Theorem 3.1.2]. Summing up over all $K \in \mathcal{T}_{h}$ we find that the integrals over all inner edges cancel out. Indeed, let $K$ and $K^{\prime}$ 
be two neighbouring cells with a common edge $E=\partial K \cap \partial K^{\prime}$. Then, for the tangential and outer normal directions we have on $E$,

$$
\mathbf{n}_{K}=-\mathbf{n}_{K^{\prime}}, \quad \mathbf{t}_{i}^{K}=-\mathbf{t}_{i}^{K^{\prime}}, \quad i=1,2,3 .
$$

Thus, apart from the sign of $\mathbf{n}_{K}$, we have the same traces of the second derivatives of $\mathbf{u}$ and of $r_{h}$ on the common edge $E$. Thus, we have shown that

$$
\begin{aligned}
\left|\left(r_{h}, \operatorname{div}\left(\mathbf{u}-i_{h} \mathbf{u}\right)\right)\right| & \leq C h^{2}|\mathbf{u}|_{3}\left\|r_{h}\right\|_{0} \\
& +\frac{1}{12} \sum_{E \subset \partial \Omega} \int_{E}\left|r_{h}\left(h_{1}^{2} \partial_{\mathbf{t}_{1} \mathbf{t}_{1}}^{2} \mathbf{u}+h_{1} h_{3} \partial_{\mathbf{t}_{1} \mathbf{t}_{3}}^{2} \mathbf{u}+h_{3}^{2} \partial_{\mathbf{t}_{3} \mathbf{t}_{3}}^{2} \mathbf{u}\right) \cdot \mathbf{n}_{E}\right| d s,
\end{aligned}
$$

from which the statement of the lemma follows by using the discrete trace inequality

$$
\left\|r_{h}\right\|_{0, E} \leq C h_{K}^{-1 / 2}\left\|r_{h}\right\|_{0, K}, \quad E \subset \partial K, \forall K \in \mathcal{T}_{h}
$$

and the continuity of the trace operator $\left.\varphi \mapsto \varphi\right|_{\partial \Omega}$ from $H^{1}(\Omega)$ in $L^{2}(\partial \Omega)$.

Consider now (3.4). Similar to [2, 4] we represent the contribution of one cell by an integral over the cell and line integrals over the edges including only the tangential derivatives of the test function $\mathbf{v}_{h}$. Integration by parts yields

$$
\int_{K} \operatorname{div}\left(\mathbf{u}-i_{h} \mathbf{u}\right) \operatorname{div} \mathbf{v}_{h} d x=\sum_{i=1}^{3} \int_{s_{i}}\left(\mathbf{u}-i_{h} \mathbf{u}\right) \cdot \mathbf{n}_{i} \operatorname{div} \mathbf{v}_{h} d s .
$$

Transformation to the reference cell, applying the Bramble-Hilbert lemma, and transforming back gives

$$
\int_{s_{i}}\left(\mathbf{u}-i_{h} \mathbf{u}\right) d s=-\frac{h_{i}^{2}}{12} \int_{s_{i}} \partial_{\mathbf{t}_{i} \mathbf{t}_{i}}^{2} \mathbf{u} d s+\mathcal{O}\left(h_{K}^{3}|\mathbf{u}|_{3, K}\right) .
$$

There are coefficients $\omega_{m n}^{K}$, with $m, n \in\{1,2\}$, such that

$$
\left.\operatorname{div} \mathbf{v}_{h}\right|_{K}=\omega_{11}^{K} \partial_{\mathbf{t}_{i}} v_{1, h}+\omega_{12}^{K} \partial_{\mathbf{t}_{i+1}} v_{1, h}+\omega_{21}^{K} \partial_{\mathbf{t}_{i}} v_{2, h}+\omega_{22}^{K} \partial_{\mathbf{t}_{i+1}} v_{2, h}
$$

The essential idea is to replace the resulting line integrals of $\partial_{\mathbf{t}_{i+1}}$ over $s_{i}$ by line integrals over $s_{i+1}$ taking into consideration that (Green's theorem)

$$
h_{i+1} \int_{s_{i}} w d s-h_{i} \int_{s_{i+1}} w d s=\frac{h_{1} h_{2} h_{3}}{2|K|} \int_{K} \partial_{\mathbf{t}_{i+2}} w d x .
$$

As a result, we obtain

$$
\begin{aligned}
\int_{s_{i}}\left(\mathbf{u}-i_{h} \mathbf{u}\right) & \cdot \mathbf{n}_{i} \operatorname{div} \mathbf{v}_{h} d s=-\frac{h_{i}^{2}}{12} \int_{s_{i}} \partial_{\mathbf{t}_{i} \mathbf{t}_{i}}^{2} \mathbf{u} \cdot \mathbf{n}_{i}\left(\omega_{11}^{K} \partial_{\mathbf{t}_{i}} v_{1, h}+\omega_{21}^{K} \partial_{\mathbf{t}_{i}} v_{2, h}\right) d s \\
& -\frac{h_{i}^{3}}{12 h_{i+1}} \int_{s_{i+1}} \partial_{\mathbf{t}_{i} \mathbf{t}_{i}}^{2} \mathbf{u} \cdot \mathbf{n}_{i}\left(\omega_{12}^{K} \partial_{\mathbf{t}_{i+1}} v_{1, h}+\omega_{22}^{K} \partial_{\mathbf{t}_{i+1}} v_{2, h}\right) d s \\
& -\frac{h_{i}^{2} h_{1} h_{2} h_{3}}{24 h_{i+1}|K|} \int_{K} \partial_{\mathbf{t}_{i+2} \mathbf{t}_{i} \mathbf{t}_{i}}^{3} \mathbf{u} \cdot \mathbf{n}_{i}\left(\omega_{12}^{K} \partial_{\mathbf{t}_{i+1}} v_{1, h}+\omega_{22}^{K} \partial_{\mathbf{t}_{i+1}} v_{2, h}\right) d x \\
& +\mathcal{O}\left(h_{K}^{3}|\mathbf{u}|_{3, K}\left|\operatorname{div} \mathbf{v}_{h}\right|_{K} \mid\right) .
\end{aligned}
$$

Now, summing over the edges $s_{i}$ of the cell $K$ and over all cells $K \in \mathcal{T}_{h}$, the line integrals cancel out, since for neighbouring cells $K$ and $K^{\prime}$,

$$
\mathbf{n}_{i}^{K}=-\mathbf{n}_{i}^{K^{\prime}}, \quad \mathbf{t}_{i}^{K}=-\mathbf{t}_{i}^{K^{\prime}}, \quad \omega_{m n}^{K}=-\omega_{m n}^{K^{\prime}}
$$


and on the boundary the tangential derivative of $\mathbf{v}_{h}$ vanishes. The sum over the integrals over $K$ gives an $\mathcal{O}\left(h^{2}\|\mathbf{u}\|_{3}\left\|\mathbf{v}_{h}\right\|_{1}\right)$ term and

$$
\left.\sum_{K \in \mathcal{T}_{h}} h_{K}^{3}|\mathbf{u}|_{3, K}\left|\operatorname{div} \mathbf{v}_{h}\right|_{K}\left|=\sum_{K \in \mathcal{T}_{h}} \frac{h_{K}^{3}}{|K|^{1 / 2}}\right| \mathbf{u}\right|_{3, K}\left\|\operatorname{div} \mathbf{v}_{h}\right\|_{0, K}=\mathcal{O}\left(h^{2}\|\mathbf{u}\|_{3}\left\|\mathbf{v}_{h}\right\|_{1}\right) .
$$

Thus, (3.4) is proven.

Moreover, we have the following estimate from interpolation theory.

Lemma 3.5. Assume $\alpha_{K} \sim h_{K}^{2}$ and $p \in H^{2}(\Omega)$. Then, it follows that

$$
\begin{aligned}
\left|\left(p-j_{h} p, \operatorname{div} \mathbf{w}_{h}\right)\right| & \leq C h^{2}\|p\|_{2}\left|\mathbf{w}_{h}\right|_{1}, & \forall \mathbf{w}_{h} & \in \mathbf{V}_{h}, \\
\left|\sum_{K \in \mathcal{T}_{h}} \alpha_{K}\left(\nabla\left(p-j_{h} p\right), \nabla r_{h}\right)_{K}\right| & \leq C h^{2}\|p\|_{2}\left\|r_{h}\right\|_{0}, & & \forall r_{h} \in Q_{h} .
\end{aligned}
$$

Finally, we need an estimate for the consistency error of the stabilized method.

Lemma 3.6. Assume $\alpha_{K} \sim h_{K}^{2}$ and $p \in H^{2}(\Omega)$. Then, it follows that

$$
\left|\sum_{K \in \mathcal{T}_{h}} \alpha_{K}\left(\nabla p, \nabla r_{h}\right)_{K}\right| \leq C h^{3 / 2}\|p\|_{2}\left\|r_{h}\right\|_{0}, \quad \forall r_{h} \in Q_{h} .
$$

Proof. Integration by parts, the continuity of the trace operator, and the discrete trace inequality (3.6) yield

$$
\begin{aligned}
\left|\sum_{K \in \mathcal{T}_{h}} \alpha_{K}\left(\nabla p, \nabla r_{h}\right)_{K}\right| & =\left|\int_{\Omega} \alpha_{K} \Delta p r_{h} d x\right|+\left|\int_{\partial \Omega} \alpha_{K} \frac{\partial p}{\partial \mathbf{n}} r_{h} d s\right| \\
& \leq C\left(h^{2}+h^{3 / 2}\right)\|p\|_{2}\left\|r_{h}\right\|_{0},
\end{aligned}
$$

from which the statement of the lemma follows.

Now, we show the supercloseness of the finite element solution $\left(\mathbf{u}_{h}, p_{h}\right)$ of the stabilized scheme (2.4) to the piecewise linear interpolant $\left(i_{h} \mathbf{u}, j_{h} p\right) \in \mathbf{V}_{h} \times Q_{h}$.

Theorem 3.7. Let $\alpha_{K} \sim h_{K}^{2}$, the mesh $\mathcal{T}_{h}$ be three-directional, and the solution $(\mathbf{u}, p)$ of (2.1) belong to $H^{3}(\Omega)^{2} \times H^{2}(\Omega)$. Then, we have the supercloseness estimate for the finite element approximation $\left(\mathbf{u}_{h}, p_{h}\right)$,

$$
\left\|||\left(\mathbf{u}_{h}-i_{h} \mathbf{u}, p_{h}-j_{h} p\right)\right\|_{A} \leq C h^{3 / 2}\left(\|\mathbf{u}\|_{3}+\|p\|_{2}\right) .
$$

Proof. From (2.1) and (2.4), we get

$$
\begin{gathered}
A_{h}\left(\left(\mathbf{u}_{h}-i_{h} \mathbf{u}, p_{h}-j_{h} p\right) ;\left(\mathbf{w}_{h}, r_{h}\right)\right)=A_{h}\left(\left(\mathbf{u}-i_{h} \mathbf{u}, p-j_{h} p\right) ;\left(\mathbf{w}_{h}, r_{h}\right)\right)+S_{h}\left(p, r_{h}\right) \\
=\left(\nabla \mathbf{u}-i_{h} \mathbf{u}, \nabla \mathbf{w}_{h}\right)-\left(p-j_{h} p, \operatorname{div} \mathbf{w}_{h}\right)+\left(r_{h}, \operatorname{div}\left(\mathbf{u}-i_{h} \mathbf{u}\right)\right) \\
+S_{h}\left(p-j_{h} p, r_{h}\right)+S_{h}\left(p, r_{h}\right) .
\end{gathered}
$$

Using the stability of the bilinear form $A_{h}$ with respect to the triple norm $\left|\|\cdot \mid\| \|_{A}\right.$ (see Lemma 2.1), we obtain

$$
\begin{aligned}
\left\|\left(\mathbf{u}_{h}-i_{h} \mathbf{u}, p_{h}-j_{h} p\right)\right\| \|_{A} \leq \frac{1}{\beta_{A}} \sup _{\left(\mathbf{w}_{h}, r_{h}\right) \in \mathbf{V}_{h} \times Q_{h}} \frac{A_{h}\left(\left(\mathbf{u}_{h}-i_{h} \mathbf{u}, p_{h}-j_{h} p\right) ;\left(\mathbf{w}_{h}, r_{h}\right)\right)}{\|\|\left(\mathbf{w}_{h}, r_{h}\right)\|\|_{A}} \\
\quad=\frac{1}{\beta_{A}} \sup _{\left(\mathbf{w}_{h}, r_{h}\right) \in \mathbf{V}_{h} \times Q_{h}} \frac{A_{h}\left(\left(\mathbf{u}-i_{h} \mathbf{u}, p-j_{h} p\right) ;\left(\mathbf{w}_{h}, r_{h}\right)\right)+S_{h}\left(p, r_{h}\right)}{\left.\left\|\left(\mathbf{w}_{h}, r_{h}\right)\right\|\right|_{A}} \\
\leq C h^{3 / 2}\left(\|\mathbf{u}\|_{3}+\|p\|_{2}\right),
\end{aligned}
$$

where the estimates in Lemmas 3.1 3.4 3.5. and 3.6 have been applied. 
In a similar way, we can show the supercloseness of the piecewise linear part $\left(\mathbf{u}_{L}, p_{L}\right)$ of the solution $\left(\mathbf{u}_{h}, p_{h}\right)$ of the LPS method for the pair of spaces

$$
\begin{aligned}
Y_{h} & =\left\{v \in H^{1}(\Omega):\left.v\right|_{K} \in P_{1}^{+}(K), \forall K \in \mathcal{T}_{h}\right\}, \\
D_{h} & =\left\{q \in L^{2}(\Omega):\left.q\right|_{K} \in P_{0}(K), \forall K \in \mathcal{T}_{h}\right\}
\end{aligned}
$$

and the choice $\alpha_{K} \sim h_{K}$ and $\alpha_{K} \sim h_{K}^{2}$, respectively. We remind the reader that the linear part is a solution of the PSPG stabilized method (2.11).

Theorem 3.8. Let $\gamma_{K}=\mathcal{O}(1), \tau_{K} \sim h_{K}^{2} b_{K}$, the mesh $\mathcal{T}_{h}$ be three-directional, and the solution $(\mathbf{u}, p)$ of (2.1) belong to $H^{3}(\Omega)^{2} \times H^{2}(\Omega)$. Then, we have the supercloseness estimate for the finite element solution $\left(\mathbf{u}_{L}, p_{L}\right)$ of (2.11) (or equivalently for the linear part of the LPS solution computed with $\alpha_{K} \sim h_{K}$ or $\alpha_{K} \sim h_{K}^{2}$ )

$$
\left|\left\|\left(\mathbf{u}_{L}-i_{h} \mathbf{u}, p_{L}-j_{h} p\right) \mid\right\|_{B} \leq C h^{3 / 2}\left(\|\mathbf{u}\|_{3}+\|p\|_{2}\right) .\right.
$$

Proof. We start with the stability of the bilinear form $B_{h}$ with respect to the triple norm $\|\mid \cdot\|_{B}$ given in Lemma 2.4, i.e.

$$
\begin{aligned}
\left\|\mid\left(\mathbf{u}_{L}-i_{h} \mathbf{u}, p_{L}-j_{h} p\right)\right\| \|_{B} & \leq \frac{1}{\beta_{B}} \sup _{\left(\mathbf{v}_{L}, q_{L}\right) \in \mathbf{V}_{L} \times Q_{L}} \frac{B_{h}\left(\left(\mathbf{u}_{L}-i_{h} \mathbf{u}, p_{L}-j_{h} p\right) ;\left(\mathbf{v}_{L}, q_{L}\right)\right)}{\left\|\mid\left(\mathbf{v}_{L}, q_{L}\right)\right\| \|_{B}} \\
& =\frac{1}{\beta_{B}} \sup _{\left(\mathbf{v}_{L}, q_{L}\right) \in \mathbf{V}_{L} \times Y_{L}} \frac{B_{h}\left(\left(\mathbf{u}-i_{h} \mathbf{u}, p-j_{h} p\right) ;\left(\mathbf{v}_{L}, q_{L}\right)\right)}{\left\|\left(\mathbf{v}_{L}, q_{L}\right)\right\| \|_{B}} .
\end{aligned}
$$

Next we use the following identity and estimate each term separately:

$$
\begin{aligned}
B_{h}\left(\left(\mathbf{u}-i_{h} \mathbf{u}, p-j_{h} p\right) ;\right. & \left.\left(\mathbf{v}_{L}, q_{L}\right)\right)=\left(\nabla\left(\mathbf{u}-i_{h} \mathbf{u}\right), \nabla \mathbf{v}_{L}\right)-\left(p-j_{h} p, \operatorname{div} \mathbf{v}_{L}\right) \\
+ & +\sum_{K \in \mathcal{T}_{h}} \gamma_{K}\left(\operatorname{div}\left(\mathbf{u}-i_{h} \mathbf{u}\right), \operatorname{div} \mathbf{v}_{L}\right)_{K}+\left(q_{L}, \operatorname{div}\left(\mathbf{u}-i_{h} \mathbf{u}\right)\right) \\
& +\sum_{K \in \mathcal{T}_{h}}\left(-\Delta\left(\mathbf{u}-i_{h} \mathbf{u}\right)+\nabla\left(p-j_{h} p\right), \tau_{K} \nabla q_{L}\right)_{K} .
\end{aligned}
$$

The first, second, and fourth terms on the right hand side can be considered as above. For the third term it follows from $\gamma_{K}=\mathcal{O}(1)$ and Lemma 3.4 that

$$
\left|\sum_{K \in \mathcal{T}_{h}} \gamma_{K}\left(\operatorname{div}\left(\mathbf{u}-i_{h} \mathbf{u}\right), \operatorname{div} \mathbf{v}_{L}\right)_{K}\right| \leq C h^{2}\|\mathbf{u}\|_{3}\left\|\mid\left(\mathbf{v}_{L}, q_{L}\right)\right\| \|_{B} .
$$

We split the last term into

$$
\begin{aligned}
\sum_{K \in \mathcal{T}_{h}}\left(-\Delta\left(\mathbf{u}-i_{h} \mathbf{u}\right)\right. & \left.+\nabla\left(p-j_{h} p\right), \tau_{K} \nabla q_{L}\right)_{K} \\
= & \sum_{K \in \mathcal{T}_{h}}\left(-\Delta\left(\mathbf{u}-i_{h} \mathbf{u}\right), \tau_{K} \nabla q_{L}\right)_{K}+\sum_{K \in \mathcal{T}_{h}}\left(\nabla\left(p-j_{h} p\right), \tau_{K} \nabla q_{L}\right)_{K} .
\end{aligned}
$$


Let $\Pi_{K}: L^{2}(K) \rightarrow P_{0}(K)$ denote the local $L^{2}$-projection onto $P_{0}(K)$. Since $\Delta i_{h} \mathbf{u}=0$ on each cell $K \in \mathcal{T}_{h}$ we have

$$
\begin{array}{r}
\left|\sum_{K \in \mathcal{T}_{h}}\left(-\Delta\left(\mathbf{u}-i_{h} \mathbf{u}\right), \tau_{K} \nabla q_{L}\right)_{K}\right| \leq \sum_{K \in \mathcal{T}_{h}}\left|\left(\Delta \mathbf{u}-\Pi_{K} \Delta \mathbf{u}, \tau_{K} \nabla q_{L}\right)_{K}\right| \\
+\left|\sum_{K \in \mathcal{T}_{h}}\left(\Pi_{K} \Delta \mathbf{u}, \tau_{K} \nabla q_{L}\right)_{K}\right| .
\end{array}
$$

The estimation of the first term on the right hand side is standard:

$$
\begin{aligned}
\sum_{K \in \mathcal{T}_{h}}\left|\left(\Delta \mathbf{u}-\Pi_{K} \Delta \mathbf{u}, \tau_{K} \nabla q_{L}\right)_{K}\right| & \leq C \sum_{K \in \mathcal{T}_{h}} h_{K}^{2}\|\mathbf{u}\|_{3, K}\left\|\tau_{K}^{1 / 2} \nabla q_{L}\right\|_{0, K} \\
& \leq C h^{2}\|\mathbf{u}\|_{3}\left\|\left(\mathbf{v}_{L}, q_{L}\right)\right\| \|_{B} .
\end{aligned}
$$

For the second the relation

$$
\left(\Pi_{K} \Delta \mathbf{u}, \tau_{K} \nabla q_{L}\right)_{K}=\frac{1}{|K|} \int_{K} \tau_{K} d x\left(\Delta \mathbf{u}, \nabla q_{L}\right)_{K}=\frac{1}{|K|} \frac{\left\|b_{K}\right\|_{0,1, K}^{2}}{\left|b_{K}\right|_{1, K}^{2}}\left(\Delta \mathbf{u}, \nabla q_{L}\right)_{K}
$$

is taken into consideration where, on a three-directional mesh,

$$
\frac{1}{|K|} \frac{\left\|b_{K}\right\|_{0,1, K}^{2}}{\left|b_{K}\right|_{1, K}^{2}}=C_{0} h_{K}^{2}
$$

with a fixed constant $C_{0}$. Integrating by parts, we get

$$
\begin{aligned}
\left|\sum_{K \in \mathcal{T}_{h}}\left(\Pi_{K} \Delta \mathbf{u}, \tau_{K} \nabla q_{L}\right)_{K}\right| & \leq C_{0} h^{2}\left|\sum_{K \in \mathcal{T}_{h}}\left(\Delta \mathbf{u}, \nabla q_{L}\right)_{K}\right| \\
& \leq C_{0} h^{2}\left\{\left\langle\Delta \mathbf{u} \cdot \mathbf{n}, q_{L}\right\rangle_{\partial \Omega}-\left(\operatorname{div} \Delta \mathbf{u}, q_{L}\right)_{\Omega}\right\} \\
& \leq C h^{3 / 2}\|\mathbf{u}\|_{3}\left\|q_{L}\right\|_{0},
\end{aligned}
$$

where in the last step the discrete trace inequality (3.6) has been applied.

Finally, we get

$$
\begin{aligned}
\left|\sum_{K \in \mathcal{T}_{h}}\left(\nabla\left(p-j_{h} p\right), \tau_{K} \nabla q_{L}\right)_{K}\right| & \leq \sum_{K \in \mathcal{T}_{h}}\left\|\tau_{K}^{1 / 2} \nabla\left(p-j_{h} p\right)\right\|_{0, K}\left\|\tau_{K}^{1 / 2} \nabla q_{L}\right\|_{0, K} \\
& \leq C \sum_{K \in \mathcal{T}_{h}} h_{K}^{2}\|p\|_{2, K}\left\|\tau_{K}^{1 / 2} \nabla q_{L}\right\|_{0, K} \\
& \leq C h^{2}\|p\|_{2}\left\|\left(\mathbf{v}_{L}, q_{L}\right)\right\| \|_{B},
\end{aligned}
$$

which completes the arguments.

From the relationship of the Standard Galerkin discretization using the MINIelement to the residual based stabilization method (2.11) with $\gamma_{K}=0$ we get the following result:

Theorem 3.9. Let $\gamma_{K}=0, \tau_{K} \sim h_{K}^{2} b_{K}$, the mesh $\mathcal{T}_{h}$ be three-directional, and the solution $(\mathbf{u}, p)$ of (2.1) belong to $H^{3}(\Omega)^{2} \times H^{2}(\Omega)$. Then, we have the supercloseness estimate for the finite element solution $\left(\mathbf{u}_{L}, p_{L}\right)$ of (2.11) or equivalently for the linear part of the MINI-element Galerkin finite element solution

$$
\left\|\left(\mathbf{u}_{L}-i_{h} \mathbf{u}, p_{L}-j_{h} p\right) \mid\right\|_{B} \leq C h^{3 / 2}\left(\|\mathbf{u}\|_{3}+\|p\|_{2}\right) .
$$


Remark 3.10. This type of supercloseness has been observed experimentally in a number of papers starting in [34, page 312]. For example the numerical results in [16. Table 4] demonstrate clearly the $3 / 2$ rate of $\left|\mathbf{u}_{L}-i_{h} \mathbf{u}\right|_{1}$.

3.2. Piecewise trilinear interpolations on brick meshes. Let us consider now the stabilized $Q_{1}-Q_{1}$ finite element on brick meshes in $\mathbb{R}^{3}$. All results are true analogously on rectangular meshes in $\mathbb{R}^{2}$. The edges of each cell $K$ are parallel to the coordinate axes; their lengths are denoted by $2 l_{K}, 2 k_{K}$, and $2 m_{K}$. We suppose that the family of meshes is shape regular; i.e., there is a constant $C$, such that

$$
C \sqrt{l_{K}^{2}+k_{K}^{2}+m_{K}^{2}} \leq \min \left\{l_{K}, k_{K}, m_{K}\right\}, \quad \forall K \in \mathcal{T}_{h} .
$$

Thus, $h$ is defined by $h:=\max _{K \in \mathcal{T}_{h}}\left\{2 \sqrt{l_{K}^{2}+k_{K}^{2}+m_{K}^{2}}\right\}$. The reference cell is given by $\hat{K}=(-1,1)^{3}$. For simplicity of notation, we will write $(x, y, z) \in K$ instead of $\left(x_{1}, x_{2}, x_{3}\right) \in K$ and $(\xi, \eta, \zeta) \in \widehat{K}$ instead of $\left(\hat{x}_{1}, \hat{x}_{2}, \hat{x}_{3}\right) \in \widehat{K}$ in this section. We introduce the nodal interpolation operator $\widehat{I}: H^{2}(\hat{K}) \rightarrow Q_{1}(\hat{K})$ with $\widehat{I} \hat{v}\left(a_{i}\right)=\hat{v}\left(a_{i}\right)$, where $a_{i}, i=1, \ldots, 8$ denote the vertices of $\hat{K}$. The interpolation $i_{h}(u)$ on an arbitrary cell $K$ is given by $\left.i_{h}(u)\right|_{K}:=\left(\widehat{I}\left(\left.u\right|_{K} \circ F_{K}\right)\right) \circ F_{K}^{-1}$, with $F_{K}$ being a bijective affine mapping from $\hat{K}$ to $K$. As usual, we apply the interpolation on vector-valued functions in a componentwise manner. The interpolation operator for the pressure $p$ is denoted by $j_{h}$ and uses the same degrees of freedom as $i_{h}$.

Lemma 3.11. Let $\mathbf{u} \in H^{3}(\Omega)^{3}$ and $i_{h} \mathbf{u}$ be the piecewise trilinear interpolant. Then, on a family of brick meshes we have

$$
\left|\left(\nabla\left(\mathbf{u}-i_{h} \mathbf{u}\right), \nabla \mathbf{w}_{h}\right)\right| \leq C h^{2}|\mathbf{u}|_{3}\left|\mathbf{w}_{h}\right|_{1} \quad \forall \mathbf{w}_{h} \in \mathbf{V}_{h} .
$$

Proof. The proof is similar to that of the supercloseness result in [25].

Lemma 3.12. Let $\alpha_{K} \sim h_{K}^{2}, p \in H^{2}(\Omega)$, and $j_{h}$ be the interpolant defined above. Then, the following estimation holds:

$$
\begin{array}{rlrl}
\left|\left(\left(p-j_{h} p\right), \operatorname{div} \mathbf{w}_{h}\right)\right| & \leq C h^{2}\|p\|_{2}\left|\mathbf{w}_{h}\right|_{1} & \forall \mathbf{w}_{h} \in \mathbf{V}_{h}, \\
\left|\sum_{K \in \mathcal{T}_{h}} \alpha_{K}\left(\nabla\left(p-j_{h} p\right), \nabla r_{h}\right)_{K}\right| & \leq C h^{2}\|p\|_{2}\left\|||\left(\mathbf{w}_{h}, r_{h}\right)\right\| \|_{A} & & \forall r_{h} \in Q_{h} .
\end{array}
$$

Proof. The estimation follows from Cauchy-Schwarz inequality and the approximation properties of the $Q_{1}$ interpolation operator.

For the estimation of the term $\left|\left(r_{h}, \operatorname{div}\left(\mathbf{u}-i_{h} \mathbf{u}\right)\right)\right|$ we need the following lemma:

Lemma 3.13. Let $\hat{u} \in H^{3}(\widehat{K})$. Then for all $\hat{r}_{h} \in Q_{1}(\widehat{K})$ we have

$$
\int_{\widehat{K}} \hat{r}_{h} \partial_{\xi}(\hat{u}-\widehat{I} \hat{u}) d \xi d \eta d \zeta=\frac{1}{3} \int_{\widehat{K}} \partial_{\xi}\left(\partial_{\xi \xi} \hat{u} \hat{r}_{h}\right) d \xi d \eta d \zeta+\mathcal{O}\left(|\hat{u}|_{3, \widehat{K}}\left\|\hat{r}_{h}\right\|_{0, \widehat{K}}\right) .
$$

Proof. Again, the Bramble-Hilbert lemma is used. For a fixed $\hat{r}_{h} \in Q_{1}(\widehat{K})$ we consider the mapping $\Psi: H^{3}(\widehat{K}) \rightarrow \mathbb{R}$,

$$
\hat{u} \mapsto \Psi(\hat{u}):=\int_{\widehat{K}} \hat{r}_{h} \partial_{\xi}(\hat{u}-\widehat{I} \hat{u}) d \xi d \eta d \zeta-\frac{1}{3} \int_{\widehat{K}} \partial_{\xi}\left(\partial_{\xi \xi} \hat{u} \hat{r}_{h}\right) d \xi d \eta d \zeta,
$$

which is obviously a linear and continuous mapping with

$$
\begin{aligned}
|\Psi(\hat{u})| & \leq C\left(\left\|\hat{r}_{h}\right\|_{0, \widehat{K}}|\hat{u}-\widehat{I} \hat{u}|_{1, \widehat{K}}+|\hat{u}|_{2, \widehat{K}}\left|\hat{r}_{h}\right|_{1, \widehat{K}}+|\hat{u}|_{3, \widehat{K}}\left\|\hat{r}_{h}\right\|_{0, \widehat{K}}\right) \\
& \leq C\left\|\hat{r}_{h}\right\|_{0, \widehat{K}}\|\hat{u}\|_{3, \widehat{K}} .
\end{aligned}
$$


We need to show that $\Psi(\hat{u})=0$ for $\hat{u} \in P_{2}(\widehat{K})$. Since $\widehat{I}$ is the $Q_{1}$ interpolation operator, and $\partial_{\xi \xi} \hat{u}=0$, for $\hat{u} \in Q_{1}(\widehat{K})$, it is sufficient to investigate $\hat{u} \in \operatorname{span}\left\{\xi^{2}, \eta^{2}, \zeta^{2}\right\}$. Since the $\xi$-derivative appears in both integrals, only $\hat{u}=\xi^{2}$ remains to analyze. With $\widehat{I} \xi^{2}=1$ we get by direct computation,

$$
\Psi\left(\xi^{2}\right)=\int_{\widehat{K}} \hat{r}_{h}(2 \xi) d \xi d \eta d \zeta-\frac{1}{3} \int_{\widehat{K}} \partial_{\xi}\left(2 \hat{r}_{h}\right) d \xi d \eta d \zeta=0 .
$$

Applying the Bramble-Hilbert lemma, we finally have

$$
|\Psi(\hat{u})| \leq C\left\|\hat{r}_{h}\right\|_{0, \widehat{K}}|\hat{u}|_{3, \widehat{K}},
$$

which is the statement of the lemma.

Remark 3.14. Analogous estimates can be shown by replacing the $\xi$-derivatives by $\eta$-derivatives and $\zeta$-derivatives, respectively.

Lemma 3.15. Let $\mathbf{u} \in H^{3}(\Omega)^{3}$ and $\mathcal{T}_{h}$ be a decomposition into bricks of uniform size $\left(l_{K}=l, k_{K}=k\right.$, and $\left.m_{K}=m\right)$. Then, we have

$$
\mid\left(r_{h}, \operatorname{div}\left(\mathbf{u}-i_{h} \mathbf{u}\right) \mid \leq C h^{3 / 2}\|\mathbf{u}\|_{3}\left\|r_{h}\right\|_{0} \quad \forall r_{h} \in Q_{h} .\right.
$$

Proof. Again, it is sufficient to consider $\left(\partial_{x}\left(u-i_{h} u\right), r_{h}\right)_{K}$. By mapping to the reference cell $\widehat{K}$ and using Lemma 3.13 we have

$$
\begin{aligned}
\int_{K} \partial_{x}\left(u-i_{h} u\right) r_{h} d x d y d z=\frac{l_{K} k_{K} m_{K}}{l_{K}} \int_{\widehat{K}} \partial_{\xi}(\hat{u}-\widehat{I} \hat{u}) \hat{r}_{h} d \xi d \eta d \zeta \\
=k_{K} m_{K}\left\{\frac{1}{3} \int_{\widehat{K}} \partial_{\xi}\left(\partial_{\xi \xi} \hat{u} \hat{r}_{h}\right) d \xi d \eta d \zeta+\mathcal{O}\left(|\hat{u}|_{3, \widehat{K}}\left\|\hat{r}_{h}\right\|_{0, \widehat{K}}\right)\right\} \\
=k_{K} m_{K}\left\{\frac{1}{3} \frac{l_{K}^{3}}{l_{K} k_{K} m_{K}} \int_{K} \partial_{x}\left(\partial_{x x} u r_{h}\right) d x d y d z+\mathcal{O}\left(|u|_{3, K}\left\|r_{h}\right\|_{0, K}\right)\right\} \\
=\frac{1}{3} l_{K}^{2} \int_{K} \partial_{x}\left(\partial_{x x} u r_{h}\right) d x d y d z+\mathcal{O}\left(h^{2}|u|_{3, K}\left\|r_{h}\right\|_{0, K}\right) .
\end{aligned}
$$

Now the integrals over the cell $K$ can be represented as the difference of integrals over opposite faces of $K$, e.g. if $S_{1}$ and $S_{2}$ are the opposite faces of $K$ belonging to the planes $x=x_{K} \pm l_{K}$, we have for any smooth function $\Lambda$,

$$
\int_{K} \partial_{x} \Lambda(x, y, z) d x d y d z=\int_{S_{1}} \Lambda\left(x_{K}+l_{K}, y, z\right) d y d z-\int_{S_{2}} \Lambda\left(x_{K}-l_{K}, y, z\right) d y d z
$$

thus

$$
\frac{1}{3} l_{K}^{2} \int_{K} \partial_{x}\left(\partial_{x x} u r_{h}\right) d x d y d z=\frac{1}{3} l_{K}^{2}\left(\int_{S_{1}}-\int_{S_{2}}\right) \partial_{x x} u r_{h} d y d z .
$$

Summing over all cells $K$, the integrals cancel out inside $\Omega$, since $r_{h}$ is continuous over the inner element faces and $l_{K}=l_{K^{\prime}}$ for neighbouring cells $K$ and $K^{\prime}$. Finally, we obtain

$$
\begin{aligned}
\left|\sum_{K \in \mathcal{T}_{h}} \int_{K} \partial_{x}\left(u-i_{h} u\right) r_{h} d x d y d z\right| & \leq C h^{2}|u|_{3}\left\|r_{h}\right\|_{0}+C h^{2} \int_{\partial \Omega}\left|\partial_{x x} u r_{h}\right| d \gamma \\
& \leq C h^{2}|u|_{3}\left\|r_{h}\right\|_{0}+C h^{2}\|u\|_{3}\left\|r_{h}\right\|_{0, \partial \Omega}
\end{aligned}
$$

by using both a global trace inequality and the discrete trace inequality (3.6). 
Lemma 3.16. Assume $\alpha_{K} \sim h_{K}^{2}$ and $p \in H^{2}(\Omega)$. Then, the estimate

$$
\left|S_{h}\left(p, r_{h}\right)\right| \leq C h^{3 / 2}\|p\|_{2}\left\|r_{h}\right\|_{0} \quad \forall r_{h} \in Q_{h}
$$

holds true for the stabilization term.

Proof. Analogous to the proof of Lemma 3.6 .

Theorem 3.17. Let $(\mathbf{u}, p) \in H^{3}(\Omega)^{3} \times H^{2}(\Omega)$ and let $i_{h}$ and $j_{h}$ be the piecewise trilinear interpolations. Then, on a familiy of uniform brick meshes we have for the LPS finite element solution,

$$
\text { || }\left(\mathbf{u}_{h}-i_{h} \mathbf{u}, p_{h}-j_{h} p\right) \mid \|_{A} \leq C h^{3 / 2}\left(\|\mathbf{u}\|_{3}+\|p\|_{2}\right) .
$$

Proof. Using Lemmas 3.11, 3.12, 3.15 and 3.16 the proof follows the line of the proof of Theorem 3.7

\section{Postprocessing And superconvergence}

4.1. Piecewise quadratic postprocessing. In this section, we define an interpolation postprocessing operator $I_{2 h}$ allowing us to improve the original finite element approximations and to obtain a superconvergence result. In contrast to the standard approach of superconvergence for the Stokes problem [17, 18, 20, 23, 25, 38, we do not need any postprocessing for the pressure because the pressure approximation itself is superconvergent:

(4.1) $\left\|p_{h}-p\right\|_{0} \leq\left\|p_{h}-j_{h} p\right\|_{0}+\left\|j_{h} p-p\right\|_{0} \leq C\left(h^{3 / 2}+h^{2}\right)\left(\|\mathbf{u}\|_{3}+\|p\|_{2}\right)$.

Here, we assume that the mesh $\mathcal{T}_{h}$ is generated from a coarse mesh $\mathcal{T}_{2 h}$ by a regular refinement (connecting the edge midpoints). Then, it is easy to see that each patch $\widetilde{K} \in \mathcal{T}_{2 h}$ consists of 4 congruent child triangles $K_{i} \in \mathcal{T}_{h}, i=1,2,3,4$, indicated in Figure 2. The $P_{2}$ postprocessing interpolation operator $I_{2 h}$ will be

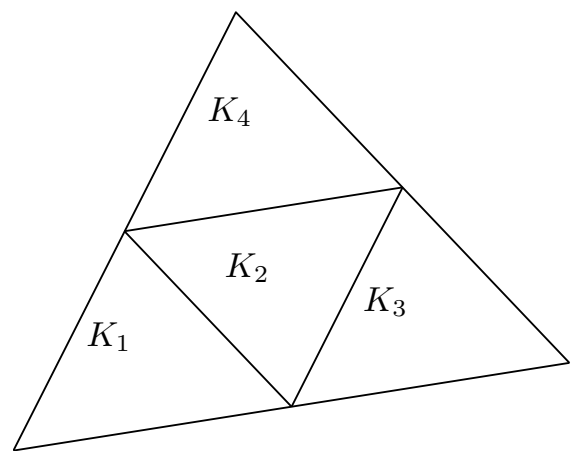

Figure 2. The patch $\widetilde{K} \in \mathcal{T}_{2 h}$ and its 4 child triangles.

locally defined by

$$
\left.I_{2 h} \mathbf{v}\right|_{\widetilde{K}}=I_{2 h}\left(\left.\mathbf{v}\right|_{\widetilde{K}}\right)
$$

where on each patch, $I_{2 h}$ coincides with the standard quadratic Lagrange nodal interpolation in the six degrees of freedom, the function values in the three vertices and the three midpoints of edges. The postprocessing interpolation operator $I_{2 h}$ satisfies the following properties. 
Lemma 4.1. For the patchwise quadratic interpolation $I_{2 h}$ and the piecewise linear interpolations $i_{h}$ and $j_{h}$ the properties

$$
\begin{array}{rlrl}
I_{2 h} i_{h} \mathbf{w} & =I_{2 h} \mathbf{w} & \forall \mathbf{w} \in C(\bar{\Omega})^{2}, \\
\left\|\left(I_{2 h} \mathbf{v}_{h}, q_{h}\right)\right\| \| & \leq C\|\|\left(\mathbf{v}_{h}, q_{h}\right) \| & & \forall\left(\mathbf{v}_{h}, q_{h}\right) \in \mathbf{V}_{h} \times Q_{h}, \\
\|\|\left(\mathbf{u}-I_{2 h} \mathbf{u}, p-j_{h} p\right)\|\| & \leq C h^{2}\left(\|\mathbf{u}\|_{3}+\|p\|_{2}\right) & \forall(\mathbf{u}, p) \in H^{3}(\Omega)^{2} \times H^{2}(\Omega)
\end{array}
$$

hold true, where $|\| \cdot|||$ denotes $\|\cdot \mid\|_{A}\left(\right.$ with $\left.\alpha_{K}=\mathcal{O}\left(h_{K}^{2}\right)\right)$ and $\|\mid \cdot\| \|_{B}$ (with $\gamma_{K}=\mathcal{O}(1)$ and $\left.\tau_{K}=\mathcal{O}\left(h_{K}^{2}\right)\right)$, respectively.

Proof. Property (4.2) is simple to see and well known. The estimate (4.4) depends on the choices of the stabilization parameters; however, for the \|\|$\cdot\|\|_{A}$-norm we have $\alpha_{K}=\mathcal{O}\left(h_{K}^{2}\right)$ and for the $\|\mid \cdot\| \|_{B}$-norm $\tau_{K}=\mathcal{O}\left(h_{K}^{2}\right)$, and $\gamma_{K}=\mathcal{O}(1)$, which is sufficient to get the second-order convergence.

For the stability (4.3) it is enough to show that

$$
\left|I_{2 h} \mathbf{v}_{h}\right|_{1, \widetilde{K}}^{2} \leq C \sum_{K \subset \widetilde{K}}\left|\mathbf{v}_{h}\right|_{1, K}^{2} \quad \forall \mathbf{v} \in \mathbf{V}_{h} .
$$

This follows by transformation onto a reference patch and norm equivalence on finite-dimensional spaces.

After constructing the postprocessing operator $I_{2 h}$, we can state the following superconvergence result.

Theorem 4.2. Assume that the postprocessing operator $I_{2 h}$ satisfies (4.2)-(4.4). Under the assumption of Theorem 3.7, we have the following superconvergence result for the case $Y_{h}=\left\{v \in H^{1}(\Omega):\left.v\right|_{K} \in P_{1}(K), \forall K \in \mathcal{T}_{h}\right\}$ and $D_{h}=\{0\}$ :

$$
\left\|\left(I_{2 h} \mathbf{u}_{h}-\mathbf{u}, p_{h}-p\right)\right\| \|_{A} \leq C h^{3 / 2}\left(\|\mathbf{u}\|_{3}+\|p\|_{2}\right)
$$

and the following superconvergence result for the piecewise linear part in the approximation space $Y_{h}=\left\{v \in H^{1}(\Omega):\left.v\right|_{K} \in P_{1}^{+}(K), \forall K \in \mathcal{T}_{h}\right\}$ and the discontinuous projection space $D_{h}=\left\{q:\left.q\right|_{K} \in P_{0}(K), \forall K \in \mathcal{T}_{h}\right\}$ :

$$
\left\|\left(I_{2 h} \mathbf{u}_{L}-\mathbf{u}, p_{L}-p\right)\right\| \|_{B} \leq C h^{3 / 2}\left(\|\mathbf{u}\|_{3}+\|p\|_{2}\right) .
$$

Proof. From (3.10) and (4.2)-(4.4), we have

$$
\begin{aligned}
&\left.\left\|\left(I_{2 h} \mathbf{u}_{h}-\mathbf{u}, p_{h}-p\right)\right\|\right|_{A} \\
& \leq\left\|\left|\left(I_{2 h}\left(\mathbf{u}_{h}-i_{h} \mathbf{u}\right), p_{h}-j_{h} p\right)\right|\right\|_{A}+\left\|\left|\left(I_{2 h} i_{h} \mathbf{u}-\mathbf{u}, j_{h} p-p\right)\right|\right\|_{A} \\
& \leq\left. C\left\|||\left(\mathbf{u}_{h}-i_{h} \mathbf{u}, p_{h}-j_{h} p\right)\right\|\right|_{A}+\left\|\left(I_{2 h} \mathbf{u}-\mathbf{u}, j_{h} p-p\right)\right\| \|_{A} \\
& \leq C h^{3 / 2}\left(\|\mathbf{u}\|_{3}+\|p\|_{2}\right) .
\end{aligned}
$$

Thus, (4.5) is shown. The estimate (4.6) follows by the same arguments.

4.2. Piecewise $d$-quadratic postprocessing. In this subsection, we construct a postprocessing interpolation operator for the rectangular or brick meshes. In the axiparallel rectangular, and brick case, no postprocessing for the pressure is needed. For the velocity, we assume similar to the triangular case that the mesh $\mathcal{T}_{h}$ was obtained from a coarse mesh $\mathcal{T}_{2 h}$ by a regular refinement. Then, each patch $\widetilde{K} \in \mathcal{T}_{2 h}$ consists of $2^{d}$ congruent child bricks $K_{i} \in \mathcal{T}_{h}, i=1,2, \ldots, 2^{d}$; see Figure 3 , 

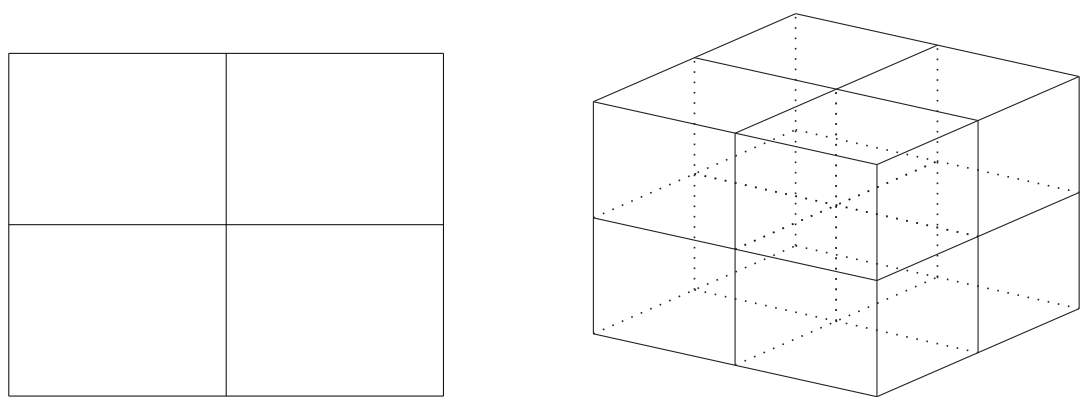

Figure 3 . The patch $\widetilde{K} \in \mathcal{T}_{2 h}$ and its child rectangles $(d=2)$ or bricks $(d=3)$.

Now, we define the $d$-quadratic interpolation operator $I_{2 h}$ locally by

$$
\left.I_{2 h} \mathbf{v}\right|_{\widetilde{K}}=I_{2 h}\left(\left.\mathbf{v}\right|_{\widetilde{K}}\right),
$$

and use on each patch $\widetilde{K}$ the $Q_{2}$ Lagrange interpolation defined by

$$
I_{2 h} \mathbf{v}\left(Z_{i}\right)=\mathbf{v}\left(Z_{i}\right)
$$

where $Z_{i}, i=1,2, \ldots, 3^{d}$ are the vertices of the child bricks belonging to the patch.

The postprocessing interpolation $I_{2 h}$ satisfies the properties (4.2)-(4.4). Thus, we have a similar superconvergence result as Theorem 4.2 for the approximation $\left(I_{2 h} \mathbf{u}_{h}, p_{h}\right)$.

Theorem 4.3. Assume that $\mathcal{T}_{h}$ is a family of axiparallel uniform rectangular or uniform brick-type meshes. Let $I_{2 h}$ be the patchwise d-quadratic interpolation. Under the assumptions of Theorem 3.17, we have the superconvergence result:

$$
\left\|\mid\left(I_{2 h} \mathbf{u}_{h}-\mathbf{u}, p_{h}-p\right)\right\| \|_{A} \leq C h^{3 / 2}\left(\|\mathbf{u}\|_{3}+\|p\|_{2}\right) .
$$

Proof. The arguments are analogous to those of the proof of Theorem 4.2 .

\section{EXtension to MORE GENERAL MESHES}

In realistic computations, we cannot always work with a three-directional mesh. Following the ideas of $[2]$ for the Poisson equation, we extend the superconvergence result valid for three-directional meshes to more general meshes.

We state first the mesh conditions. Two adjacent triangles (sharing a common edge) are said to form an $\mathcal{O}\left(h^{1+\rho}\right)(\rho>0)$ approximate parallelogram if the lengths of any two opposite edges differ only by $\mathcal{O}\left(h^{1+\rho}\right)$.

Definition 5.1. The triangulation $\mathcal{T}_{h}=\mathcal{T}_{1, h} \cup \mathcal{T}_{2, h}$ is said to satisfy condition $(\rho, \sigma)$ if there exist positive constants $\rho$ and $\sigma$ such that every two adjacent triangles inside $\mathcal{T}_{1, h}$ form an $O\left(h^{1+\rho}\right)$ parallelogram and

$$
\bar{\Omega}_{1, h} \cup \bar{\Omega}_{2, h}=\bar{\Omega}, \quad\left|\Omega_{2, h}\right|=O\left(h^{\sigma}\right), \quad \bar{\Omega}_{i, h}=\bigcup_{K \in \mathcal{T}_{i, h}} \bar{K}, \quad i=1,2 .
$$

The condition $(\rho, \sigma)$ is a reasonable condition in practice and can be satisfied by most automatically generated meshes. In fact, $\Omega_{2 h}$ contains only exceptional elements, and these are relatively few in number. We set $\beta:=\min \left(\rho, \frac{1}{2}, \frac{\sigma}{2}\right)$. Then, we have the following estimates for meshes satisfying the condition $(\rho, \sigma)$. 
Lemma $5.2(23,37])$. Assume that $\mathbf{u} \in\left(H^{3}(\Omega) \cap W^{2, \infty}(\Omega)\right)^{2}$. Then, we have the following estimate for meshes satisfying the condition $(\rho, \sigma)$ :

$$
\left|\left(\nabla\left(\mathbf{u}-i_{h} \mathbf{u}\right), \nabla \mathbf{w}_{h}\right)\right| \leq C h^{1+\beta}\left(\|\mathbf{u}\|_{3}+\|\mathbf{u}\|_{2, \infty}\right)\left|\mathbf{w}_{h}\right|_{1} \quad \forall \mathbf{w}_{h} \in \mathbf{V}_{h}
$$

Lemma 5.3. Assume that $\mathbf{u} \in\left(H^{3}(\Omega) \cap W^{2, \infty}(\Omega)\right)^{2}$. Then, we have the following estimate for meshes satisfying the condition $(\rho, \sigma)$ :

$$
\begin{aligned}
\left|\left(r_{h}, \operatorname{div}\left(\mathbf{u}-i_{h} \mathbf{u}\right)\right)\right| \leq C h^{1+\beta}\left(\|\mathbf{u}\|_{3}+\|\mathbf{u}\|_{2, \infty}\right)\left\|r_{h}\right\|_{0} \quad \forall r_{h} \in Q_{h}, \\
\left|\left(\operatorname{div}\left(\mathbf{u}-i_{h} \mathbf{u}\right), \operatorname{div} \mathbf{v}_{h}\right)\right| \leq C h^{1+\beta}\left(\|\mathbf{u}\|_{3}+\|\mathbf{u}\|_{2, \infty}\right)\left|\mathbf{w}_{h}\right|_{1} \quad \forall \mathbf{v}_{h} \in \mathbf{V}_{h} .
\end{aligned}
$$

Proof. As in the proof of Lemma 3.4. we obtain by integration by parts,

$$
\begin{aligned}
& \left(r_{h}, \operatorname{div}\left(\mathbf{u}-i_{h} \mathbf{u}_{h}\right)\right) \\
& =\frac{1}{12} \sum_{K \in \mathcal{T}_{h}} \int_{K} r_{h} \operatorname{div}\left(h_{1}^{2} \partial_{\mathbf{t}_{1} \mathbf{t}_{1}}^{2} \mathbf{u}+h_{1} h_{3} \partial_{\mathbf{t}_{1} \mathbf{t}_{3}}^{2} \mathbf{u}+h_{3}^{2} \partial_{\mathbf{t}_{3} \mathbf{t}_{3}}^{2} \mathbf{u}\right) d x+R \\
& \quad-\frac{1}{12} \sum_{K \in \mathcal{T}_{h}} \int_{\partial K} r_{h}\left(h_{1}^{2} \partial_{\mathbf{t}_{1} \mathbf{t}_{1}}^{2} \mathbf{u}+h_{1} h_{3} \partial_{\mathbf{t}_{1} \mathbf{t}_{3}}^{2} \mathbf{u}+h_{3}^{2} \partial_{\mathbf{t}_{3} \mathbf{t}_{3}}^{2} \mathbf{u}\right) \cdot \mathbf{n}_{K} d s
\end{aligned}
$$

where again, the first and the second term can be bounded by $C h^{2}\|\mathbf{u}\|_{3}\left\|r_{h}\right\|_{0}$. In the third term, we replace the derivatives in the tangential directions $\mathbf{t}_{1}$ and $\mathbf{t}_{3}$ by derivatives in the tangential direction $\mathbf{t}_{2}$ and the normal direction $\mathbf{n}_{2}$, respectively. With $\Theta_{i}$ the angle opposite to $s_{i}, i=1,2,3$, we have

$$
h_{1}^{2} \partial_{\mathbf{t}_{1} \mathbf{t}_{1}}^{2} \mathbf{u}+h_{1} h_{3} \partial_{\mathbf{t}_{1} \mathbf{t}_{3}}^{2} \mathbf{u}+h_{3}^{2} \partial_{\mathbf{t}_{3} \mathbf{t}_{3}}^{2} \mathbf{u}=F \partial_{\mathbf{t}_{2} \mathbf{t}_{2}}^{2} \mathbf{u}+G \partial_{\mathbf{t}_{2} \mathbf{n}_{2}}^{2} \mathbf{u}+H \partial_{\mathbf{n}_{2} \mathbf{n}_{2}}^{2} \mathbf{u}
$$

where

$$
\begin{aligned}
& F=h_{1}^{2} \cos ^{2} \Theta_{3}+h_{1} h_{3} \cos \Theta_{1} \cos \Theta_{3}+h_{3}^{2} \cos ^{2} \Theta_{1}, \\
& G=h_{1} \sin \Theta_{3}\left(h_{3} \cos \Theta_{1}-h_{1} \cos \Theta_{3}\right), \\
& H=h_{1}^{2} \sin ^{2} \Theta_{3} .
\end{aligned}
$$

Let us split the set of all edges into three different classes. $\mathcal{E}_{1}$ is the set of inner edges $E$ such that the two adjacent triangles sharing $E$ form an $O\left(h^{1+\rho}\right)$ approximate parallelogram, $\mathcal{E}_{2}$ is the set of remaining inner edges, and $\mathcal{E}_{3}$ is the set of all boundary edges. Consider now an edge $E=\partial K \cap \partial K^{\prime} \in \mathcal{E}_{1}$. Then, we have $\left|h_{1}-h_{1}^{\prime}\right| \leq C h_{2}^{1+\rho}$, $h_{E}=h_{2}=h_{2}^{\prime}$, and $\left|h_{3}-h_{3}^{\prime}\right| \leq C h_{2}^{1+\rho}$, from which the estimates

$$
\left|F-F^{\prime}\right| \leq C h_{2}^{2+\rho}, \quad\left|G-G^{\prime}\right| \leq C h_{2}^{2+\rho}, \quad\left|H-H^{\prime}\right| \leq C h_{2}^{2+\rho}
$$

follow by geometric considerations. Since $\mathbf{n}_{K}=-\mathbf{n}_{K^{\prime}}$, the sum of integrals over the common edge $E$ can be estimated as

$$
\begin{gathered}
\left|\int_{E} r_{h}\left(\left(F-F^{\prime}\right) \partial_{\mathbf{t}_{2} \mathbf{t}_{2}}^{2} \mathbf{u}+\left(G-G^{\prime}\right) \partial_{\mathbf{t}_{2} \mathbf{n}_{2}}^{2} \mathbf{u}+\left(H-H^{\prime}\right) \partial_{\mathbf{n}_{2} \mathbf{n}_{2}}^{2} \mathbf{u}\right) \cdot \mathbf{n}_{K} d s\right| \\
\leq C h_{E}^{2+\rho}\left\|r_{h}\right\|_{0,1, E}\|\mathbf{u}\|_{2, \infty, K \cup K^{\prime}}, \quad E \in \mathcal{E}_{1} .
\end{gathered}
$$


Furthermore, we have the following estimates:

$$
\begin{gathered}
\left|\int_{E} r_{h}\left(\left(F-F^{\prime}\right) \partial_{\mathbf{t}_{2} \mathbf{t}_{2}}^{2} \mathbf{u}+\left(G-G^{\prime}\right) \partial_{\mathbf{t}_{2} \mathbf{n}_{2}}^{2} \mathbf{u}+\left(H-H^{\prime}\right) \partial_{\mathbf{n}_{2} \mathbf{n}_{2}}^{2} \mathbf{u}\right) \cdot \mathbf{n}_{K} d s\right| \\
\leq C h_{E}^{2}\left\|r_{h}\right\|_{0,1, E}\|\mathbf{u}\|_{2, \infty, K \cup K^{\prime}}, \quad E \in \mathcal{E}_{2}, \\
\left|\int_{E} r_{h}\left(F \partial_{\mathbf{t}_{2} \mathbf{t}_{2}}^{2} \mathbf{u}+G \partial_{\mathbf{t}_{2} \mathbf{n}_{2}}^{2} \mathbf{u}+H \partial_{\mathbf{n}_{2} \mathbf{n}_{2}}^{2} \mathbf{u}\right) \cdot \mathbf{n}_{K} d s\right| \\
\leq C h_{E}^{2}\left\|r_{h}\right\|_{0,1, E}\|\mathbf{u}\|_{2, \infty, K}, \quad E \in \mathcal{E}_{3} .
\end{gathered}
$$

The estimate of the sums over the three types of edges is based on the discrete trace inequality

$$
h_{E}\left\|r_{h}\right\|_{0,1, E} \leq C|K|^{1 / 2}\left\|r_{h}\right\|_{0, K} \quad \forall r_{h} \in Q_{h}, E \subset \partial K,
$$

from which we get by summation

$$
\sum_{E \in \mathcal{E}_{i}} h_{E}\left\|r_{h}\right\|_{0,1, E} \leq C\left(\sum_{\substack{K \in \mathcal{T}_{h} \\ \exists E \in \mathcal{E}_{i}: \partial K \cap E \neq \emptyset}}|K|\right)^{1 / 2}\left\|r_{h}\right\|_{0} .
$$

The discrete trace inequality (5.7) follows from scaling properties, the trace inequality on the reference cell, and the equivalence of norms in finite-dimensional spaces. Applying (5.7) to (5.4)-(5.6) we end up with

$$
\begin{aligned}
& \left|\sum_{E \in \mathcal{E}_{1}} \int_{E} \cdots d s\right| \leq C h^{1+\rho}\left(\sum_{K \in \mathcal{T}_{1, h}}|K|\right)^{1 / 2}\|\mathbf{u}\|_{2, \infty, \Omega}\left\|r_{h}\right\|_{0} \leq C h^{1+\rho}\|\mathbf{u}\|_{2, \infty, \Omega}\left\|r_{h}\right\|_{0}, \\
& \left|\sum_{E \in \mathcal{E}_{2}} \int_{E} \cdots d s\right| \leq C h\left(\sum_{K \in \mathcal{T}_{2, h}}|K|\right)^{1 / 2}\|\mathbf{u}\|_{2, \infty, \Omega}\left\|r_{h}\right\|_{0} \leq C h^{1+\sigma / 2}\|\mathbf{u}\|_{2, \infty, \Omega}\left\|r_{h}\right\|_{0}, \\
& \left|\sum_{E \in \mathcal{E}_{3}} \int_{E} \cdots d s\right| \leq C h\left(\sum_{\bar{K} \cap \Gamma \neq \emptyset}|K|\right)^{1 / 2}\|\mathbf{u}\|_{2, \infty, \Omega}\left\|r_{h}\right\|_{0} \leq C h^{3 / 2}\|\mathbf{u}\|_{2, \infty, \Omega}\left\|r_{h}\right\|_{0} .
\end{aligned}
$$

Thus, (5.2) is proven. Along the same lines, (5.3) can be shown.

These meshes are not generated by a regular refinement. Therefore a reasonable postprocessing is given by recovery methods for linear finite elements as already used for second-order elliptic problems; see e.g. 39, 40, 43. We define a recovery operator $G_{h}: \mathbf{V}_{h} \rightarrow\left(Y_{h} \times Y_{h}\right)^{2}$, where $Y_{h}=\left\{v \in H^{1}(\Omega):\left.v\right|_{K} \in P_{1}(K), \forall K \in \mathcal{T}_{h}\right\}$. The piecewise linear function $G_{h} \mathbf{u}_{h}$ is an approximation to the gradient of the exact solution $\nabla \mathbf{u}$ constructed by the finite element solution $\mathbf{u}_{h}$. This operator has the following properties:

$$
\begin{array}{rlrl}
\left\|G_{h} \mathbf{v}_{h}\right\|_{0} & \leq C\left\|\mathbf{v}_{h}\right\|_{1}, & & \forall \mathbf{v}_{h} \in \mathbf{V}_{h}, \\
\left\|G_{h} \mathbf{u}-\nabla \mathbf{u}\right\|_{0} & \leq C h^{2}\|\mathbf{u}\|_{3}, & & \forall \mathbf{u} \in H^{3}(\Omega)^{2}, \\
G_{h} \mathbf{u} & =G_{h}\left(i_{h} \mathbf{u}\right), & \forall \mathbf{u} \in H^{3}(\Omega)^{2} .
\end{array}
$$


Theorem 5.4. Let the solution $(\mathbf{u}, p)$ of (2.1) belong to $\left(H^{3}(\Omega) \cap W^{2, \infty}(\Omega)\right)^{2} \times$ $H^{2}(\Omega)$. Then, we have the following supercloseness on meshes satisfying the condition $(\rho, \sigma)$ :

$$
\|\|\left(\mathbf{u}_{h}-i_{h} \mathbf{u}, p_{h}-j_{h} p\right) \|\left.\right|_{A} \leq C h^{1+\beta}\left(\|\mathbf{u}\|_{3}+\|\mathbf{u}\|_{2, \infty}+\|p\|_{2}\right)
$$

for the $P_{1}$ case. Furthermore, for the $P_{1}^{+}$case the linear part $\left(\mathbf{u}_{L}, p_{L}\right)$ of the discrete solution satisfies

$$
\left\|\mid\left(\mathbf{u}_{L}-i_{h} \mathbf{u}, p_{L}-j_{h} p\right)\right\|_{B} \leq C h^{1+\beta}\left(\|\mathbf{u}\|_{3}+\|\mathbf{u}\|_{2, \infty}+\|p\|_{2}\right) .
$$

For the recovered gradients the superconvergence results

$$
\begin{aligned}
&\left\|G_{h} \mathbf{u}_{h}-\nabla \mathbf{u}\right\|_{0}+\left\|p_{h}-p\right\|_{0} \leq C h^{1+\beta}\left(\|\mathbf{u}\|_{3}+\|\mathbf{u}\|_{2, \infty}+\|p\|_{2}\right), \\
&\left\|G_{h} \mathbf{u}_{L}-\nabla \mathbf{u}\right\|_{0}+\left\|p_{L}-p\right\|_{0} \leq C h^{1+\beta}\left(\|\mathbf{u}\|_{3}+\|\mathbf{u}\|_{2, \infty}+\|p\|_{2}\right)
\end{aligned}
$$

hold true.

Proof. The supercloseness results (5.11) and (5.12) follow from the stability of the underlying bilinear forms, the Lemmas $5.2,5.3,3.5$, and 3.6, in the same way as shown in Section 3.1

Combining (5.8)-(5.10) and (5.11), we have

$$
\begin{aligned}
\left\|G_{h} \mathbf{u}_{h}-\nabla \mathbf{u}\right\|_{0}+\left\|p_{h}-p\right\|_{0} \leq & \left\|G_{h} \mathbf{u}_{h}-G_{h}\left(i_{h} \mathbf{u}\right)\right\|_{0}+\left\|G_{h}\left(i_{h} \mathbf{u}\right)-G_{h} \mathbf{u}\right\|_{0} \\
& +\left\|G_{h} \mathbf{u}-\nabla \mathbf{u}\right\|_{0}+\left\|p_{h}-p\right\|_{0} \\
\leq & C\left\|\mathbf{u}_{h}-i_{h} \mathbf{u}\right\|_{1}+C h^{2}\left(\|\mathbf{u}\|_{3}+\|p\|_{2}\right) \\
\leq & C h^{1+\beta}\left(\|\mathbf{u}\|_{3}+\|\mathbf{u}\|_{2, \infty}+\|p\|_{2}\right) .
\end{aligned}
$$

The estimate (5.14) can be proven similarly.

In fact, the condition $(\rho, \sigma)$ is very general. For a general domain, we start with a coarse mesh and use a regular refinement. Then, the resulting family of meshes satisfies the condition $(\rho, \sigma)$.

\section{NumericAl TESTS}

In this section we present numerical results for solving the Stokes problem by the stabilized method (2.4) for the $P_{1}$ case and the $P_{1}^{+}$case, respectively.

Example 6.1. Let $\Omega=(0,1)^{2}$. We consider the Stokes problem

$$
-\Delta \mathbf{u}+\nabla p=\mathbf{f}, \quad \operatorname{div} \mathbf{u}=0 \quad \text { in } \Omega, \quad \mathbf{u}=\mathbf{g} \quad \text { on } \partial \Omega,
$$

where the right hand side $\mathbf{f}$ and the inhomogeneous Dirichlet boundary condition g are chosen such that

$$
\mathbf{u}=\left(\begin{array}{c}
\sin (x) \sin (y) \\
\cos (x) \cos (y)
\end{array}\right), \quad p=2 \cos (x) \sin (y)-2 \sin (1)(1-\cos (1))
$$

is the solution.

We compute the finite element solution on uniform triangular meshes of a regular pattern obtained by successive regular refinement of an initial coarse mesh. The mesh on level 1 is shown in Figure 4.

In the following, we evaluate the results of our computation by considering the $H^{1}$ seminorm of the velocity error and the $L^{2}$ norm of the pressure error. We also compute the $H^{1}$ seminorm error of the postprocessed velocity $I_{2 h} \mathbf{u}_{h}$. The numerical 
results confirm the theoretically predicted convergence rates; see Figure 5 for the $P_{1}$ case and Figure 6 for the $P_{1}^{+}$case, respectively.

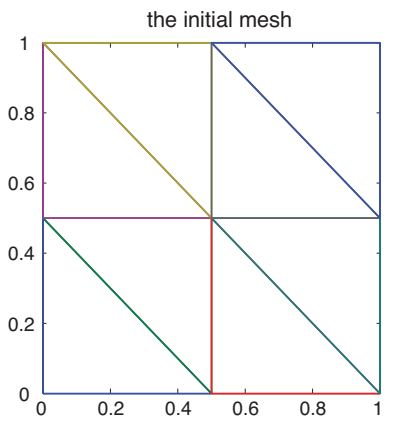

Figure 4. The initial uniform mesh.
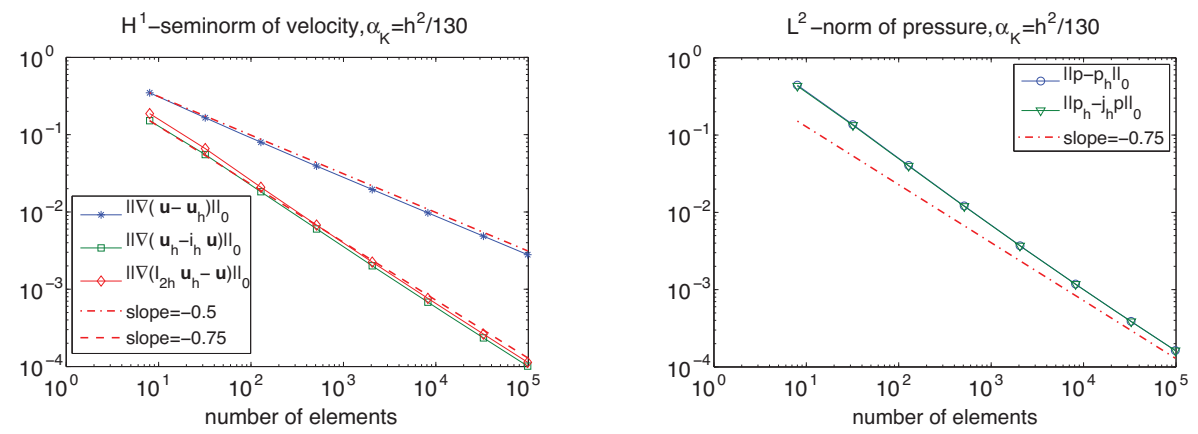

FiguRe 5 . Velocity and pressure error for the $P_{1}$ case on threedirectional meshes.
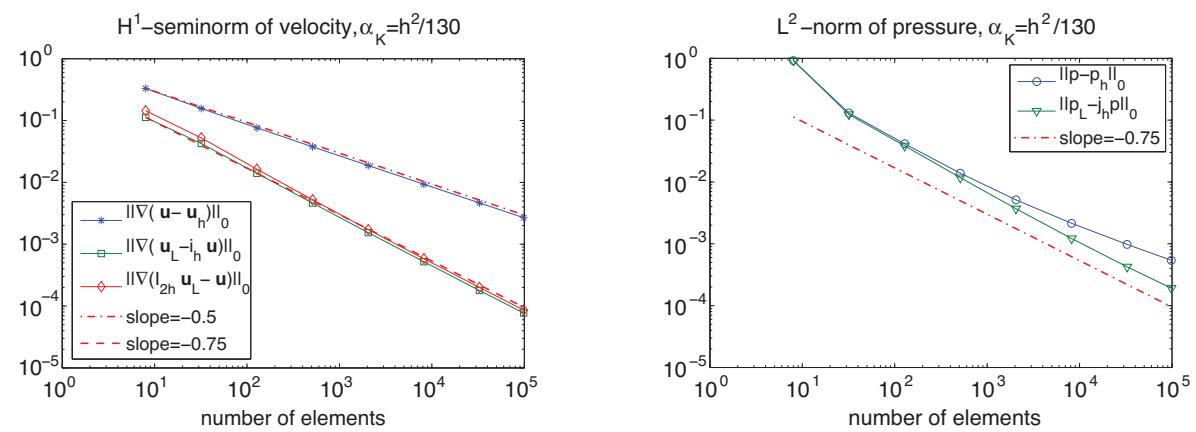

Figure 6 . Velocity and pressure error for the $P_{1}^{+}$case on threedirectional meshes. 
Example 6.2. In this example, we solve the Stokes problem (6.1) on a unit circle where the right hand side $\mathbf{f}$ and the inhomogeneous Dirichlet boundary condition $\mathrm{g}$ are chosen such that

$$
\mathbf{u}=\left(\begin{array}{c}
\sin (x) \sin (y) \\
\cos (x) \cos (y)
\end{array}\right), \quad p=2 \cos (x) \sin (y)
$$

is the exact solution.

In this example, the domain $\Omega$ cannot be triangulated by a family of threedirectional meshes. Here, we used the Delaunay triangulation to produce an initial coarse mesh. Then, a family of meshes is generated by successive regular refinement. Figure 7 shows the initial mesh and the refined mesh on level 4. In Figures 8 and 9 the errors for the velocity and the pressure are shown for the $P_{1}$ case and the $P_{1}^{+}$ case, respectively. We observe superconvergence also over this type of successively refined meshes. Note that, the technique of 21] has been used to carry out the postprocessing.

We also considered an automatic generated family of meshes (Delaunay triangulation separately on each mesh level). Even in this case we observed some (less pronounced) superconvergence properties.
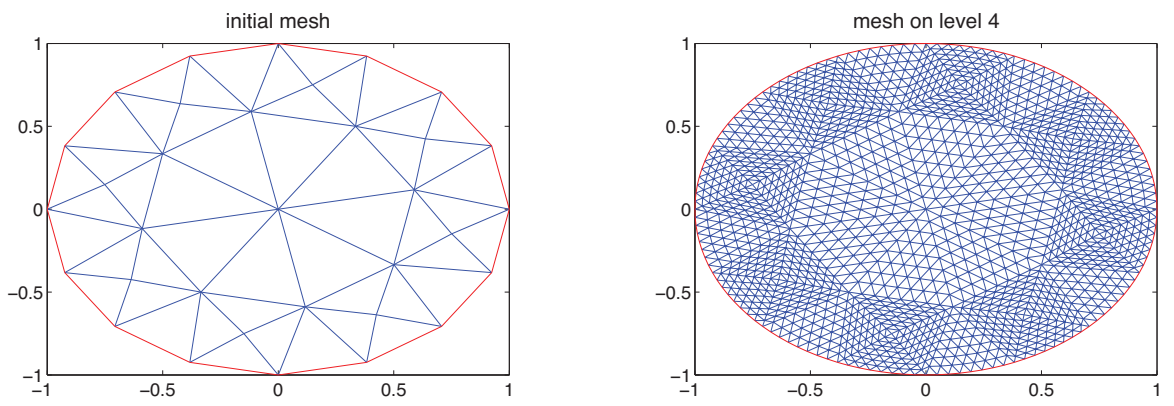

Figure 7 . The meshes on level 0 and level 4 for a regular refinement.
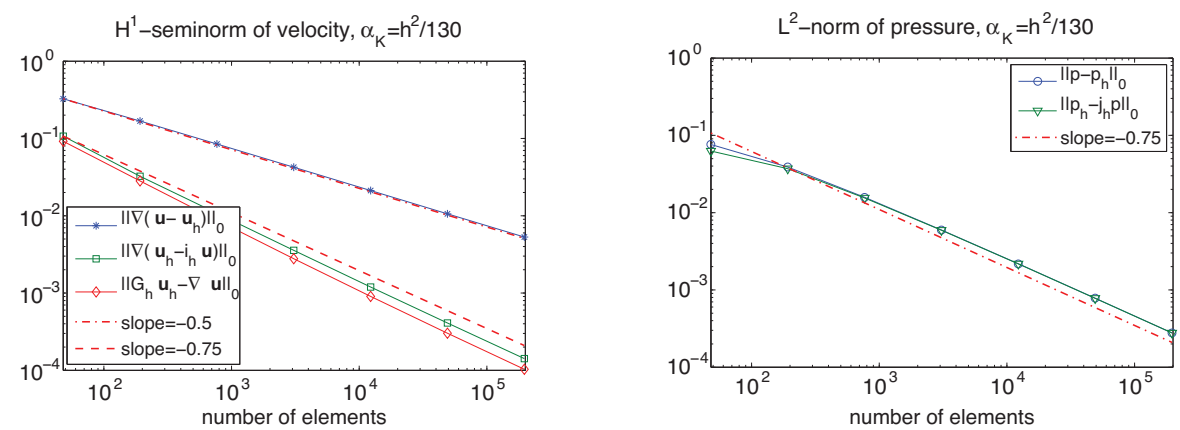

FiguRE 8. Velocity and pressure error for the regular refinement $P_{1}$ case. 

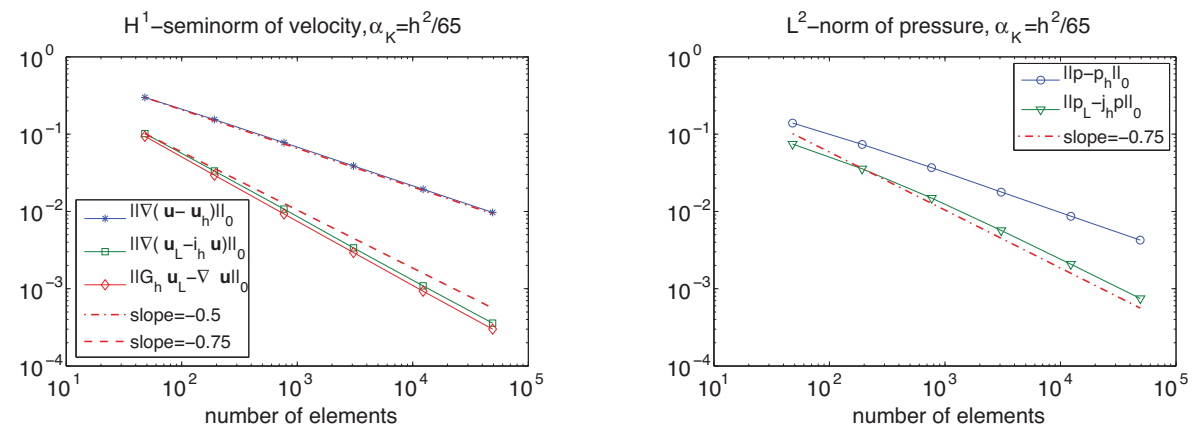

Figure 9. Velocity and pressure error for the regular refinement $P_{1}^{+}$case.

\section{ACKNOWLEDGEMENT}

The research has been partially supported by the BMBF-Project SimParTurS under the grant 03TOPAA1. The second author gratefully acknowledges the support from the Chinese Academy of Sciences during his stay at the State Key Laboratory of Scientific and Engineering Computing, Chinese Academy of Sciences, Beijing.

\section{REFERENCES}

1. D. N. Arnold, F. Brezzi, and M. Fortin, A stable finite element for the Stokes equation, Calcolo 21 (1984), 337-344. MR799997 (86m:65136)

2. R.E. Bank and J. Xu, Asymptotically exact a posteriori error estimators. I. Grids with superconvergence, SIAM J. Numer. Anal. 41 (2003), no. 6, 2294-2312 (electronic). MR 2034616 (2004k:65194)

3. R. Becker and M. Braack, A finite element pressure gradient stabilization for the Stokes equations based on local projections, Calcolo 38 (2001), no. 4, 173-199. MR.1890352(2002m:65112)

4. H. Blum, Q. Lin, and R. Rannacher, Asymptotic error expansion and Richardson extrapolation for linear finite elements, Numer. Math. 49 (1986), 11-37. MR847015 (87m:65172)

5. M. Braack and E. Burman, Local projection stabilization for the Oseen problem and its interpretation as a variational multiscale method, SIAM J. Numer. Anal. 43 (2006), no. 6, 2544-2566. MR2206447 (2007a:65139)

6. F. Brezzi and M. Fortin, Mixed and hybrid finite element methods, Springer Series in Computational Mathematics, vol. 15, Springer-Verlag, New York, 1991. MR.1115205 (92d:65187)

7. F. Brezzi and J. Pitkäranta, On the stabilization of finite element approximations of the Stokes equations, Efficient solutions of elliptic systems (Kiel, 1984), Notes Numer. Fluid Mech., vol. 10, Vieweg, Braunschweig, 1984, pp. 11-19. MR804083 (86j:65147)

8. C. Chen and Y. Huang, High accuracy theory of finite element methods, Hunan Science and Technology Press, Hunan, China, 1995.

9. P.G. Ciarlet, The finite element method for elliptic problems, North-Holland Publishing Co., Amsterdam, 1978, Studies in Mathematics and its Applications, Vol. 4. MR0520174 $(58 \# 25001)$

10. L.P. Franca and S.L. Frey, Stabilized finite element methods: II. The incompressible NavierStokes equations, Comput. Methods Appl. Mech. Engrg. 99 (1992), no. 2/3, 209-233. MR.1186727 (93i:76055)

11. S. Ganesan, G. Matthies, and L. Tobiska, Local projection stabilization of equal order interpolation applied to the Stokes problem, Math. Comp. 77 (2008), no. 264, 2039-2060. MR2429873 (2009h:65182)

12. V. Girault and P.A. Raviart, Finite element methods for Navier-Stokes equations, Springer Series in Computational Mathematics, vol. 5, Springer-Verlag, Berlin, 1986, Theory and algorithms. MR851383 (88b:65129) 
13. P. Hansbo and A. Szepessy, A velocity-pressure streamline diffusion method for the incompressible Navier-Stokes equations, Comput. Methods Appl. Mech. Engrg. 84 (1990), 175-192. MR.1087615 (91k:76109)

14. T.J.R. Hughes, L.P. Franca, and M. Balestra, A new finite element formulation for computational fluid dynamics. V: Circumventing the Babuška-Brezzi condition: A stable PetrovGalerkin formulation of the Stokes problem accomodating equal-order interpolations, Comput. Methods Appl. Mech. Engrg. 59 (1986), 85-99. MR868143 (89j:76015d)

15. __ Errata: "A new finite element formulation for computational fluid dynamics. $V$. Circumventing the Babuška-Brezzi condition: A stable Petrov-Galerkin formulation of the Stokes problem accommodating equal-order interpolations", Comput. Methods Appl. Mech. Engrg. 62 (1987), no. 1, 111. MR889303 (89j:76015e)

16. Y. Kim and S. Lee, Modified Mini finite element for the Stokes problem in $\mathbb{R}^{2}$ or $\mathbb{R}^{3}$, Adv. Comput. Math. 12 (2000), 261-272. MR1745116(2001h:65145)

17. J. Lin and Q. Lin, Extrapolation of the Hood-Taylor elements for the Stokes problem, Adv. Comput. Math. 22 (2005), no. 2, 115-123. MR2126582 (2005m:65274)

18. Q. Lin, J. Li, and A. Zhou, A rectangle test for the Stokes problem, Proceedings of Systems Science \& Systems Engineering, Great Wall (H. K.) Culture Publish Co., 1991, pp. 240-241.

19. Q. Lin and J. Lin, Finite element methods: Accuracy and improvement, China Sci. Tech. Press, 2005.

20. Q. Lin and J. Pan, Global superconvergence for rectangular elements in the Stokes problem, Proceedings of Systems Science \& Systems Engineering, Great Wall (H. K.) Culture Publish Co., 1991, pp. 371-378.

21. Q. Lin and H. Xie, A type of finite element gradient recovery method based on vertex-edge-face interpolation: The recovery technique and superconvergence property, Tech. report, LSEC, CAS, Beijing, 2009.

22. Q. Lin and J. Xu, Linear finite elements with high accuracy, J. Comput. Math. 3 (1985), no. 2, 115-133. MR854355 (87k:65141)

23. Q. Lin and N. Yan, The construction and analysis of high efficiency finite element methods, HeBei University Publishers, 1995.

24. Q. Lin and Q. Zhu, Preproceesing and postprocessing for the finite element method (in Chinese), Shanghai Scientific \& Technical Press, 1994.

25. G. Matthies, P. Skrzypacz, and L. Tobiska, Superconvergence of a 3D finite element method for stationary Stokes and Navier-Stokes problems, Numer. Methods Partial Differential Equations 21 (2005), no. 4, 701-725. MR.2140564 (2006a:65167)

26. _ A unified convergence analysis for local projection stabilisations applied to the Oseen problem, Math. Model. Numer. Anal. M2AN 41 (2007), no. 4, 713-742. MR2362912 (2008j:65201)

27. K. Nafa and A.J. Wathen, Local projection stabilized Galerkin approximations for the generalized Stokes problem, Comput. Methods Appl. Mech. Engrg. 198 (2009), 877-883. MR2498529

28. L.A. Oganesyan and L.A. Rukhovets, Study of the rate of convergence of variational difference schemes for second-order elliptic equations in a two-dimensional field with smooth boundary, Zh. Vychisl. Mat. Mat. Fiz. 9 (1969), 1102-1120 (Russian). MR0295599 (45:4665)

29. F. Schieweck, Uniformly stable mixed hp-finite elements on multilevel adaptive grids with hanging nodes, Math. Model. Numer. Anal. M2AN 42 (2008), 493-505. MR2423796 (2009d:65180)

30. L.R. Scott and S. Zhang, Finite element interpolation of nonsmooth functions satisfying boundary conditions, Math. Comput. 54 (1990), no. 190, 483-493. MR1011446 (90j:65021)

31. T.E. Tezduyar, S. Mittal, S.E. Ray, and R. Shih, Incompressible flow computations with stabilized bilinear and linear equal order interpolation velocity pressure elements, Comput. Methods Appl. Mech. Eng. 95 (1992), 221-242.

32. L. Tobiska and G. Lube, A modified streamline diffusion method for solving the stationary Navier-Stokes equations, Numer. Math. 59 (1991), 13-29. MR1103751(92c:65141)

33. L. Tobiska and R. Verfürth, Analysis of a streamline diffusion finite element method for the Stokes and Navier-Stokes equations, SIAM J. Numer. Anal. 33 (1996), 107-127. MR1377246 (97e:65133)

34. R. Verfürth, A posteriori error estimators for the Stokes equations, Numer. Math. 55 (1989), 309-325. MR993474 (90d:65187) 
35. L.B. Wahlbin, Superconvergence in Galerkin finite element methods, Lecture Notes in Mathematics, vol. 1605, Springer-Verlag, Berlin, 1995. MR1439050 (98j:65083)

36. J. Wang and X. Ye, Superconvergence of finite element approximations for the Stokes problem by projection methods, SIAM J. Numer. Anal. 39 (2001), no. 3, 1001-1013 (electronic). MR 1860454 (2002g:65151)

37. J. Xu and Z. Zhang, Analysis of recovery type a posteriori error estimators for mildly structured grids, Math. Comp. 73 (2004), no. 247, 1139-1152 (electronic). MR 2047081 (2005f:65141)

38. N. Yan, Superconvergence analysis and a posteriori error estimation in finite element methods, Science Press, Beijing, 2008.

39. Z. Zhang, Recovery Techniques in Finite Element Methods, Adaptive Computations: Theory and Algorithms (Tao Tang and Jinchao Xu, eds.), Mathematics Monograph Series 6, Science Publisher, 2007, pp. 333-412.

40. Z. Zhang and A. Naga, A new finite element gradient recovery method: Superconvergence property, SIAM J. Sci. Comput. 26 (2005), no. 4, 1192-1213 (electronic). MR2143481 (2006d:65137)

41. A. Zhou, An analysis of some high accuracy finite element methods for hyperbolic problems, SIAM J. Numer. Anal. 39 (2001), no. 3, 1014-1028. MR.1860455(2002g:65154)

42. A. Zhou and Q. Lin, Optimal and superconvergence estimates of the finite element method for a scalar hyperbolic equation, Acta Math. Sci. (English Ed.) 14 (1994), no. 1, 90-94. MR 1280088 (95c:65161)

43. O.C. Zienkiewicz and J.Z. Zhu, A simple error estimator and adaptive procedure for practical engineering analysis, Internat. J. Numer. Methods Engrg. 24 (1987), no. 2, 337-357. MR 875306 (87m:73055)

44. - The superconvergent patch recovery and a posteriori error estimates. I. The recovery technique, Internat. J. Numer. Methods Engrg. 33 (1992), no. 7, 1331-1364. MR1161557 (93c:73098)

Institute for Analysis and Computational Mathematics, Otto-von-Guericke UniverSity Magdeburg, Postfach 4120, D-39016 Magdeburg, Germany

E-mail address: hagen.eichel@st.ovgu.de

Institute for Analysis and Computational Mathematics, Otto-von-Guericke UniverSity Magdeburg, Postfach 4120, D-39016 Magdeburg, Germany

E-mail address: tobiska@ovgu.de

LSeC, Academy of Mathematics and Systems Science, Chinese Academy of Sciences, BEIJING 100080, China

Current address: Institute for Analysis and Computational Mathematics, Otto-von-Guericke University Magdeburg, Postfach 4120, D-39016 Magdeburg, Germany

E-mail address: hhxie@lsec.cc.ac.cn 